\title{
Proposal power and majority rule in multilateral bargaining with costly recognition
}

\author{
Huseyin Yildirim* \\ Department of Economics, Duke University, Durham, NC 27708, USA \\ Received 21 June 2005; final version received 5 July 2006 \\ Available online 8 September 2006
}

\begin{abstract}
This paper studies a sequential bargaining model in which agents expend efforts to be the proposer. In equilibrium, agents' effort choices are influenced by the prize and cost effects. The (endogenous) prize is the difference between the residual surplus an agent obtains when he is the proposer and the payment he expects to receive when he is not. Main results include: (1) under the unanimity voting rule, two agents with equal marginal costs propose with equal probabilities, regardless of their time preferences; (2) under a nonunanimity rule, however, the more patient agent proposes with a greater probability; (3) while, under the unanimity rule, the social cost decreases in group heterogeneity, it can increase under a nonunanimity rule; and (4) when agents are identical, the unanimity rule is socially optimal.

(C) 2006 Elsevier Inc. All rights reserved.
\end{abstract}

JEL classification: C70; D72

Keywords: Sequential bargaining; Proposal power; Majority rule; Rent-seeking contests; Recognition

\section{Introduction}

Many economic and political settings involve multilateral bargaining in which a group of agents negotiate over the allocation of some surplus. Such settings range from two nations' negotiating over a disputed territory, to legislators' deciding on the distribution of funds across states, parties' negotiating over the formation of a government in a multiparty parliamentary system, various divisions of an organization negotiating over scarce resources, and existing members of an international club, e.g., NAFTA, EU or WTO, negotiating the terms of accession for a candidate country. While in some cases agreement requires the unanimous

\footnotetext{
* Fax: +1 9196848974

E-mail address: yildirh@econ.duke.edu.
} 
approval of all interested parties, in others, the approval by a subset of agents is sufficient to implement a specific allocation. For instance, whereas a unanimous approval is needed for the enlargement programs of most international clubs or an organization's budget plan, the approval by a simple majority is sufficient to reach a legislative decision or to form a coalitional government.

Building on Rubinstein's [33] pioneering work, an elegant theoretical literature has emerged on multilateral (sequential) bargaining generating testable predictions about the equilibrium outcomes in a wide variety of environments. A key prediction of this literature is the presence of the "proposal power" in that the agent who proposes how to allocate the surplus receives a disproportionate share. ${ }^{1}$ Thus, understanding how the proposal power is gained and distributed among negotiating parties is crucial in understanding the allocation of surplus, and the parties' payoffs. With some exceptions discussed below, the extant literature assumes an exogenous "recognition process" that selects the proposer according to certain rigid institutional and organizational procedures. For instance, whereas the alternating offer models a la Rubinstein [33] allow agents to take turns making proposals, a subsequent generalization introduced by Binmore [5] endows agents with a fixed probability of recognition. Absent such rigid procedures, however, agents might take costly measures to tip the proposal power in their favor. Examples abound. In organizations such as a university, a public agency, or a corporation, the allocation of scarce resources is often the outcome of active negotiations between different units, rather than external rules, and the share each unit receives is mostly determined by its power gained through costly activities. (See, e.g., [14,18,30].) At international negotiations such as the ones between Pakistan and India, and those between Greek and Turkish Cypriots over disputed territories, involved nations have often lobbied other nations to gain support for their proposals. Finally, in mediated bargaining, negotiating parties need to convince and educate the mediator about their demands, which frequently require hiring experts and professionals who can process information and present the case more effectively on their behalf. $^{2}$

The objective of this paper is to endogenize the recognition process by letting agents compete to be the proposer. Aside from generating proposal power as an equilibrium outcome, this will also allow us to link the incentives to propose to agents' characteristics such as their time preferences and cost efficiency as well as to the institutional and organizational variables such as the voting rule and the number of agents. Several interesting issues arise from the analysis. Regarding agents' characteristics, do more patient agents have a lesser incentive to propose? Does competition for the proposal power become more intense in a more homogenous group? Can the cost of recognition ever outweigh the benefits of proposal power in equilibrium? Regarding the institutional and organizational variables, what is the role of voting rules on the competition for and the distribution of proposal power?

The formal model builds on the Baron and Ferejohn [1-3] framework, where a group of agents wants to divide a fixed surplus among themselves. Instead of assigning a fixed probability of recognition, however, I assume, as in the rent-seeking literature, that agents expend (unproduc-

\footnotetext{
1 There is growing empirical evidence that confirms this prediction. For instance, Knight [19] uncovers that representatives affiliated with the Congressional transportation committee have used their proposal power to secure more project spending for their districts than other representatives.

${ }^{2}$ It is important that the recognition of an agent to propose be interpreted in a broader sense to include cases in which the agent does not literally propose but the proposal put forward, say by a mediator, is closest to his.
} 
tive) efforts to be recognized and win the rents associated with the proposal power. ${ }^{3,4}$ In particular, before each round of bargaining, agents simultaneously choose their effort levels, which stochastically determine the proposer. The greater one's effort is, the more likely he is to propose. As is common in the literature on sequential bargaining, I assume agents adopt stationary strategies, and use the stationary subgame perfect equilibrium (SSPE) as the solution concept throughout.

A brief preview of my main findings is as follows. Consistent with Baron and Ferejohn [1-3] and most bargaining models with complete information, there are no delays in equilibrium, since the proposer secures the consent of a minimal "winning coalition" by including in the allocation those agents with the "cheapest" votes. Unlike these models, however, inefficiencies do arise in equilibrium owing to unproductive efforts that agents expend to be recognized. The extent of these inefficiencies depends on the (net) prize each agent hopes to earn by being the proposer and his marginal cost of effort. The prize, which is endogenous to the bargaining process, is the difference between the residual surplus an agent claims when he is the proposer and the payment he expects to receive when he is not. It is well-known in the literature that under the unanimity rule, all agents receive the same prize irrespective of their time preferences, ${ }^{5}$ and thus I find that two agents with equal marginal costs exert the same effort, and propose with equal probabilities in equilibrium. The prize from proposing does, however, vary across players, when agreement requires less than unanimous approval. In particular, players with more expensive votes expect to earn a greater prize, because such players are less likely to be included in winning coalitions. I show that, all else equal, more patient players possess more expensive votes and consequently exert a greater effort to propose. Despite gaining the proposal power, however, they can end up being worse off than less patient ones, which is in sharp contrast to the case with the unanimity rule under which patient agents are always better off in equilibrium.

Next, I investigate the extent of the social cost generated by the resources wasted during the recognition process, and how it changes with the group heterogeneity and the voting rule in place. Under the unanimity rule, the social cost decreases as the group becomes more heterogeneous in the sense of a mean-preserving spread. To see this, note that such a group is likely to contain agents with both more and less expensive votes than does the original group. However, as explained in detail in Section 3, the net effect is an increase in the overall price of votes. Under the unanimity rule, since every agent's affirmative vote is needed, this implies that the heterogeneity reduces the prize from proposing, and hence the incentives to propose. In a sense, the presence of "tougher" bargainers in the group helps reduce wasteful activities. A similar line of argument, however, reveals that the social cost can actually increase in a more heterogenous group, when agreement requires a less than unanimous approval. This is because unlike the unanimity rule, the most expensive votes need not be bought out, raising the prize from proposing and consequently intensifying the competition to propose.

\footnotetext{
${ }^{3}$ There is a large literature on rent-seeking behavior in which a group of agents expends effort to win a given prize. See, e.g., Nitzan [25] for a survey.

${ }^{4}$ To my knowledge, the only other paper that allows players to expend effort to propose is Evans [13]. Extending the coalitional bargaining framework introduced by Chatterjee et al. [8], Evans shows that the pure strategy subgame perfect payoff set coincides with the core. A noteworthy feature of his model is that no equilibrium efforts are expended in pure strategies.

Also related to my work is the literature, e.g., Perez-Castrillo and Wettstein [28,29], which tries to implement the Shapley value through a noncooperative game involving bidding for the right to propose. I elaborate on the link between my work and this literature in the concluding section, when I discuss possible extensions.

${ }^{5}$ See, for instance, Merlo and Wilson [23].
} 
Given the equilibrium social cost, a natural question is how voting rules affect it. Restricting attention to symmetric agents, I find that the social cost is lower, the closer the voting rule is to the unanimity, implying the optimality of the unanimity rule. The intuition is that as the voting rule becomes more inclusive, the prize from proposing gets smaller, reducing incentives to exert effort. But this, in turn, benefits agents.

The rest of the paper is organized as follows. The next section lays out the elements of the model. Section 3 analyzes the case with the unanimity rule. Section 4 relaxes this assumption and considers general voting rules. Section 5 investigates the impact of voting rules on equilibrium inefficiencies. Section 6 presents an extension where recognition probability also has an intrinsic component and demonstrates the robustness of the main results. Finally, Section 7 offers concluding remarks. All proofs are contained in an Appendix.

\section{The model}

Consider a situation in which a group of agents decides how to allocate a perfectly divisible surplus of unit size among themselves. Let $N \equiv\{1,2, \ldots, n\}$ denote the set of agents, and $S \equiv\left\{\left(s_{1}, s_{2}, \ldots, s_{n}\right) \mid \forall i \in N, s_{i} \geqslant 0\right.$ and $\left.\sum_{i} s_{i} \leqslant 1\right\}$ denote the set of feasible allocations, where $s_{i}$ is the share agent $i$ receives. Assume that each agent is risk neutral, and that he discounts the future returns and costs by $\delta_{i} \in[0,1)$. The interaction among agents is modeled as a sequential bargaining game with complete information, where the proposer needs the consent of $k$ players (including himself) for his proposal to be agreed upon. This " $k$-majority" voting procedure captures a variety of bargaining environments. At one extreme, $k=n$ refers to the unanimity rule granting each player veto power. For a standard two-player setting, the unanimity rule follows by definition. At the other extreme, $k=1$ refers to a situation where the proposer becomes a one-period dictator. When $n$ is odd, $k=\frac{n+1}{2}$ refers to the simple majority rule.

Timing and information structure: At the beginning of period $t=0$, players simultaneously exert efforts. Let $x_{i, t} \geqslant 0$ and $C_{i}\left(x_{i, t}\right)$ represent player $i$ 's effort and its cost, respectively. For simplicity, I assume $C_{i}\left(x_{i, t}\right)=c_{i} x_{i, t}$. Once efforts are chosen, player $i$ is recognized with probability $p_{i}\left(x_{i, t}, \mathbf{x}_{-i, t}\right)$ to make a proposal from the set $S$. Each player then decides whether to "accept" or "reject" the proposal according to a prespecified order. If at least $k$ players accept, then the proposal is implemented and the game ends. Otherwise, the proposal is rejected, and the game repeats itself at $t=1$ except that players choose their efforts again and a player is recognized. ${ }^{6}$ This process continues until an allocation generates the required number of votes, whereupon player $i$ receives a payoff, $\delta_{i}^{t} s_{i, t}-\sum_{t^{\prime}=0}^{t} \delta_{i}^{t^{\prime}} C_{i}\left(x_{i, t^{\prime}}\right)$. If no agreement is ever reached, then all players are assumed to receive $-\sum_{t^{\prime}=0}^{\infty} \delta_{i}^{t^{\prime}} C_{i}\left(x_{i, t^{\prime}}\right)$.

Recognition probabilities: Given that our analysis builds on the literature on contests, e.g., Nitzan [25], the structure on the recognition probability closely follows the one on the "contest success function" in that literature. In particular, I make the following assumptions:

A.1. Let $p_{i}\left(x_{i}, \mathbf{x}_{-i}\right):\left[0, \frac{1}{c_{i}}\right] \times \prod_{j \neq i}\left[0, \frac{1}{c_{j}}\right] \rightarrow[0,1]$ be player $i$ 's recognition probability with these properties:

(a) $p_{i}(\mathbf{x})$ is twice continuously differentiable in all arguments.

\footnotetext{
${ }^{6}$ In Section 6, I briefly discuss an extension in which effort has a persistent effect on recognition.
} 
(b) $\sum_{i} p_{i}(\mathbf{x})=1, p_{i}(\mathbf{0})=\frac{1}{n}$; and $p_{i}\left(x_{i}, \mathbf{0}\right)=1, \frac{\partial}{\partial x_{i}} p_{i}\left(0, \mathbf{x}_{-i}\right)>\frac{\partial}{\partial x_{i}} p_{i}\left(x_{i}, \widehat{\mathbf{x}}_{-i}\right)$ for $x_{i}>0$ and $\mathbf{x}_{-i} \geqslant \widehat{\mathbf{x}}_{-i} \cdot{ }^{7}$

(c) (Diminishing marginal returns) $\frac{\partial}{\partial x_{i}} p_{i}(\mathbf{x})>0, \frac{\partial^{2}}{\partial x_{i}^{2}} p_{i}(\mathbf{x})<0$; and $\frac{\partial}{\partial x_{j}} p_{i}(\mathbf{x})<0, \frac{\partial^{2}}{\partial x_{j} \partial x_{k}} p_{i}(\mathbf{x})$ $>0$ for $j, k \neq i$.

(d) (Symmetry) $p_{i}\left(x_{i}, \mathbf{x}_{-i}\right)=p_{i}\left(x_{i}, \widehat{\mathbf{x}}_{-i}\right)$, where $\widehat{\mathbf{x}}_{-i}$ is any permutation of $\mathbf{x}_{-i}$.

Part (b) provides tie-breaking and boundary conditions in case of no effort by some agent(s). Part (c) says that player $i$ 's recognition probability increases in his own effort and decreases in rivals', both at a decreasing rate. Part (d) is an anonymity property implying that recognition depends only on players' efforts, and not on their identities. In particular, if two players exert the same effort, then their recognition probabilities must be equal.

In what follows, I will have to frequently impose more structure on the recognition probabilities, in particular to guarantee the uniqueness of equilibrium efforts and payoffs, and also to characterize the social cost. In such cases, I assume ${ }^{8}$

A.2. Let $p_{i}(\mathbf{x}):\left[0, \frac{1}{c_{i}}\right] \times \prod_{j \neq i}\left[0, \frac{1}{c_{j}}\right] \rightarrow[0,1]$ be player $i$ 's recognition probability, such that

$$
p_{i}(\mathbf{x})= \begin{cases}\frac{f\left(x_{i}\right)}{\sum_{j} f\left(x_{j}\right)} & \text { if } \mathbf{x} \neq \mathbf{0} \\ \frac{1}{n} & \text { if } \mathbf{x}=\mathbf{0}\end{cases}
$$

where $f(0)=0, f^{\prime}\left(x_{i}\right)>0, f^{\prime \prime}\left(x_{i}\right) \leqslant 0$, and the elasticity of the "production function", $f$, defined as $\varepsilon\left(x_{i}\right) \equiv \frac{f^{\prime}\left(x_{i}\right) x_{i}}{f\left(x_{i}\right)}$ is weakly decreasing.

Note that A. 2 holds for many well-known functions used in the rent-seeking literature, e.g., $x_{i}^{\alpha}$ with $\alpha \in(0,1], 1-e^{-x_{i}}$, and $\ln \left(1+x_{i}\right)$. Note also that the properties of $f\left(x_{i}\right)$ imply $\varepsilon\left(x_{i}\right) \in(0,1]$. However, these properties are not sufficient for $\varepsilon\left(x_{i}\right)$ to be weakly decreasing. A counterexample is $f\left(x_{i}\right)=x_{i}+\ln \left(1+x_{i}\right)$.

As alluded to in the Introduction, there are three noteworthy features of our model. First, it endogenizes the recognition process. Absent strict institutional and organizational procedures that assign the proposal rights, it is conceivable that agents will expend resources to claim the rents associated with proposal power. Here, I simply follow the rent-seeking literature and assume that agents compete for these rents. Indeed, at its core, this paper combines the two literatures on multilateral bargaining with an exogenous recognition rule and the rent-seeking contests, admitting each as a special case. On the one hand, when $\mathbf{x}=\mathbf{0}$, the model reduces to the previous models of sequential bargaining with an exogenous recognition process. ${ }^{9}$ On the other hand, when $\mathbf{x} \neq \mathbf{0}$, and players put no weight on the future or acceptance of a proposal requires only one vote, the model coincides with the standard rent-seeking model, in which a group of players

\footnotetext{
${ }^{7}$ I define the ordering relation " $\geqslant$ " to be such that for any two vectors $\mathbf{a}, \mathbf{b} \in R^{m}, \mathbf{a} \geqslant \mathbf{b}$ if $a_{j} \geqslant b_{j}$ for all $j \in\{1, \ldots, m\}$. Similarly, I will say $\mathbf{a}>\mathbf{b}$ if $a_{j}>b_{j}$ for all $j \in\{1, \ldots, m\}$.

${ }^{8}$ This functional form is commonly assumed in the contest literature, and one axiomatic derivation can be found in Skaperdas [35]. I could easily introduce heterogeneity in $f$; but this would be equivalent to cost heterogeneity and hence not change the qualitative results.

${ }^{9}$ I consider an extension that elaborates on this point in Section 6.
} 
compete to win a fixed prize. In general, though, agents will compete to win the endogenous and possibly different prizes from proposing that are determined through the bargaining process. ${ }^{10}$

Second, our model assumes that effort has no long-lasting effect on recognition. This is a reasonable approximation in cases where negotiating parties need to renew the support for their proposals. For instance, in mediated bargaining, parties might have to repeat their efforts for the recognition of their proposals, if the mediator is short-lived. ${ }^{11}$ Third, players may differ both in their discount factors (as is usual in many bargaining settings) and in their marginal costs. I will show that in general, the two sources of heterogeneity will have different implications.

I now describe the solution concept. Let $H_{t}$ be the history of the game that contains the identity of proposers, proposals that have been made, and actions taken up to period $t$. In period $t$, player $i$ exerts effort $x_{i, t}\left(H_{t}\right)$ and takes action $a_{i, t}\left(H_{t}\right)$ such that

$$
a_{i, t}\left(H_{t}\right) \in \begin{cases}S & \text { if player } i \text { is the proposer, } \\ \{\text { accept, reject }\} & \text { otherwise. }\end{cases}
$$

A strategy $\xi_{i}$ for player $i$ describes a sequence of efforts and actions, $\left\{x_{i, t}\left(H_{t}\right), a_{i, t}\left(H_{t}\right)\right\}_{t=1}^{t=\infty}$. A strategy profile, $\xi \equiv\left(\xi_{1}, \ldots, \xi_{n}\right)$, is stationary if it is time and history independent. Furthermore, a strategy profile is a subgame perfect equilibrium (SPE) if it constitutes a Nash equilibrium in each period, and it is a SSPE, henceforth "equilibrium", if it is both stationary and subgame perfect. Intuitively, an SSPE calls for the same actions in each continuation game followed by rejection of an offer on the table. There are three main reasons why I focus on the SSPE. First, unlike the bilateral bargaining game studied by Rubinstein [33], multilateral bargaining games entail a plethora of SPE (see, e.g., $[2,3,17,36])$. However, the stationarity restriction often dramatically reduces the equilibrium set and hence is widely used in the literature (see, e.g., [2,3,8,10,11,15,22,23]). Second, an SSPE may entail the least "complexity" in certain bargaining games similar to the one analyzed here [4] and therefore serve as a natural focal point. Third, it is analytically tractable.

To fix intuition behind costly recognition and voting rules, I first examine the case with the unanimity rule, and relax this assumption in Section 4.

\section{Unanimity rule}

Suppose a proposal requires the unanimous approval of all agents in order to be implemented, i.e., $k=n$. Let $v_{i}$ denote the expected equilibrium payoff for player $i$ before efforts are chosen and the identity of the proposer is revealed. When not the proposer, a player will accept a proposal so long as he is allocated an amount no less than his continuation payoff. Since the proposer maximizes his own share of the surplus, each nonproposer will be offered exactly this payoff, resulting in no equilibrium delays. Thus, to determine the effort level, a player $i$ needs to take two possibilities into account. First, with probability $p_{i}(\mathbf{x})$, he is recognized to propose, in which case he offers the continuation values to all other agents, i.e., $\delta_{j} v_{j}$ for all $j \neq i$, while retaining the rest of the surplus, $1-\sum_{j \neq i} \delta_{j} v_{j}$, for himself. Second, with probability $1-p_{i}(\mathbf{x})$, someone else is recognized, in which case he expects to receive his continuation value, $\delta_{i} v_{i}$. Overall, the

\footnotetext{
${ }^{10}$ A strand of the rent-seeking literature, e.g., Leininger [20], and Nti [26], considers cases in which agents attach different exogenous values to the prize.

11 There are other bargaining situations in which agents' recognition might also have a persistent component. I investigate this possibility in Section 6.
} 
expected equilibrium payoff for player $i$ satisfies the following dynamic program:

$$
v_{i}=\max _{x_{i} \in\left[0, \frac{1}{c_{i}}\right]}\left\{p_{i}\left(x_{i}, \mathbf{x}_{-i}\right)\left[1-\sum_{j \neq i} \delta_{j} v_{j}\right]+\left[1-p_{i}\left(x_{i}, \mathbf{x}_{-i}\right)\right] \delta_{i} v_{i}-c_{i} x_{i}\right\},
$$

or, equivalently

$$
v_{i}=\max _{x_{i} \in\left[0, \frac{1}{c_{i}}\right]}\left\{p_{i}\left(x_{i}, \mathbf{x}_{-i}\right)\left[1-\sum_{j} \delta_{j} v_{j}\right]+\delta_{i} v_{i}-c_{i} x_{i}\right\} .
$$

Taking the derivative of the terms inside the brackets yields

$$
\text { FOC }: \frac{\partial p_{i}\left(x_{i}, \mathbf{x}_{-i}\right)}{\partial x_{i}}\left[1-\sum_{j} \delta_{j} v_{j}\right]-c_{i} \leqslant 0 \quad\left(=0 \text { if } x_{i}>0\right) .
$$

The condition in (3) reveals that player $i$ 's effort choice is influenced by the (net) prize he expects to receive upon being recognized and his marginal cost. The size of the prize is $\pi_{i}^{n} \equiv 1-\sum_{j} \delta_{j} v_{j}$, where superscript of $\pi_{i}^{n}$ refers to the unanimity rule. Somewhat surprisingly, this prize is equal across agents, irrespective of their discount factors. ${ }^{12}$ The intuition is that when recognized to be the proposer, player $i$ receives $1-\sum_{j \neq i} \delta_{j} v_{j}$ after buying out the votes of all other agents.

However, since player $i$ 's vote is not bought out by another player in this case, he also forgoes his continuation value, $\delta_{i} v_{i}$. The net prize for player $i$ is thus the residual surplus after he pays for all votes including his own.

Finding the equilibrium under the unanimity rule amounts to finding a pair of $(\mathbf{x}, \mathbf{v})$ that satisfies (2) for all agents. To distinguish these prize and cost effects, I first consider the case where players differ only in their discount factors.

Proposition 1. Suppose $p_{i}(\mathbf{x})$ satisfies A.1 and $c_{i}=c$ for all $i \in N$. Then, under the unanimity rule, there exists a unique equilibrium such that for all $i, j \in N$

- $x_{i}=x_{j}>0$.

- $p_{i}(\mathbf{x})=p_{j}(\mathbf{x})=\frac{1}{n}$.

- $\left(1-\delta_{i}\right) v_{i}=\left(1-\delta_{j}\right) v_{j}$.

The proofs of this and subsequent results are relegated to an appendix. As explained above, the first two parts of Proposition 1 follow because all agents expect to receive the same prize from proposing, and, given equal marginal costs, they exert the same effort. This also means each agent is equally likely to propose in equilibrium. The last part of Proposition 1 confirms our intuition that the more patient agent receives a higher equilibrium payoff, by simply being able to reject unfavorable offers.

When agents also differ in their marginal costs of effort, the results in Proposition 1 are modified in an intuitive way. However, we somewhat forgo the generality by relying on A.2.

12 This fact has been also observed in the multilateral bargaining literature, e.g., Merlo and Wilson [23]. 
Proposition 2. Suppose $p_{i}(\mathbf{x})$ satisfies A.2. Then, under the unanimity rule, there exists a unique equilibrium with these properties: $x_{l}>0$ for some $l \in N$, and if $c_{i}<c_{j}$ for some $i, j \in N$, then

- $x_{i} \geqslant x_{j}$

- $p_{i}(\mathbf{x}) \geqslant p_{j}(\mathbf{x})$

- $\left(1-\delta_{i}\right) v_{i} \geqslant\left(1-\delta_{j}\right) v_{j}$,

where strict inequality holds whenever $x_{i}>0$.

According to Proposition 2, agents take advantage of being more efficient by expending a greater effort to propose. Again, this is so regardless of their discount factors. The last part of Proposition 2 implies that all else being equal, i.e., the discount factors are the same, the more efficient agent receives a greater payoff. However, it is possible that such an agent may end up with a lower payoff if he is sufficiently impatient relative to others. This is intuitive because both cost efficiency and patience are sources of bargaining power. I illustrate this point with a simple example. ${ }^{13}$

Example 1. Suppose there are two agents, $i=1,2$ and $p_{i}(\mathbf{x})$ satisfies A.2 with $f\left(x_{i}\right)=x_{i}$. It is easy to verify that in equilibrium, both agents exert strictly positive efforts such that total costs are equal and given by

$$
c_{i} x_{i}=\frac{\left(1-\delta_{i}\right)\left(1-\delta_{j}\right)}{\frac{c_{i}}{c_{j}}\left(1-\delta_{i}\right)+\frac{c_{j}}{c_{i}}\left(1-\delta_{j}\right)+2\left(1-\delta_{i}\right)\left(1-\delta_{j}\right)} .
$$

This implies agent $i$ is recognized with probability $p_{i}=\frac{c_{j}}{c_{i}+c_{j}}$. Moreover, agent $i$ 's expected share from surplus ${ }^{14}$ is

$$
\bar{s}_{i}=\frac{\left(1-\delta_{j}\right)\left[1+\frac{c_{i}}{c_{j}}\left(1-\delta_{i}\right)\right]}{\left(1-\delta_{j}\right)+\left(\frac{c_{i}}{c_{j}}\right)^{2}\left(1-\delta_{i}\right)+2 \frac{c_{i}}{c_{j}}\left(1-\delta_{i}\right)\left(1-\delta_{j}\right)} .
$$

Subtracting the expression in (4) from (5), we find $i$ 's expected payoff:

$$
v_{i}=\frac{\left(1-\delta_{j}\right)}{\left(1-\delta_{j}\right)+\left(\frac{c_{i}}{c_{j}}\right)^{2}\left(1-\delta_{i}\right)+2 \frac{c_{i}}{c_{j}}\left(1-\delta_{i}\right)\left(1-\delta_{j}\right)} .
$$

Inspecting (4) and (5), it is clear that as agent $i$ 's relative cost advantage increases, i.e., $\frac{c_{i}}{c_{j}}$ gets smaller, he proposes with a greater probability, and grabs a larger share from the surplus. Although this might mean a higher total cost, his (net) expected payoff in (6) increases. The positive effect of $i$ 's relative cost advantage may, however, lose its strength with his relative impatience. In particular, as $\delta_{j} \rightarrow 1, v_{i} \rightarrow 0$, regardless of $i$ 's cost advantage.

An important aspect of our model is that agents engage in socially wasteful activities to increase their chances of recognition. Not surprisingly, the extent of social cost depends on parameters of

\footnotetext{
13 The details of this and other examples are available from the author upon request.

${ }^{14}$ Clearly, the expected share is $\bar{s}_{i}=p_{i}\left[1-\delta_{j} v_{j}\right]+\left(1-p_{i}\right) \delta_{i} v_{i}$.
} 
the bargaining environment, especially on the group heterogeneity and the number of agents. To see the impact of each parameter, I first compute the equilibrium social cost, $S C$.

Lemma 1. Suppose $p_{i}(\mathbf{x})$ satisfies A.2. Then, under the unanimity rule, the social cost is given by

$$
S C=\sum_{i} c_{i} x_{i}=\frac{1-\sum_{i} \bar{p}_{i}(\mathbf{x})}{1-\sum_{i} \bar{p}_{i}(\mathbf{x})+\sum_{i} \frac{\bar{p}_{i}(\mathbf{x})}{1-\delta_{i}},}
$$

where $\bar{p}_{i}(\mathbf{x}) \equiv\left(1-\varepsilon\left(x_{i}\right)\right) p_{i}(\mathbf{x})+\varepsilon\left(x_{i}\right)\left(p_{i}(\mathbf{x})\right)^{2}$.

Using the concept of the mean-preserving spread as the measure of heterogeneity in the group with respect to the parameter in question, ${ }^{15}$ the following result records the properties of the social cost.

Proposition 3. Suppose $p_{i}(\mathbf{x})$ satisfies A.2 and the voting rule is unanimity.

- Given $c_{i}=c$ for all $i \in N, S C$ decreases with the group heterogeneity in $\delta_{i}$ 's.

- Given $\delta_{i}=\delta$ for all $i \in N$, and $f\left(x_{i}\right)=x_{i}$, SC decreases with the group heterogeneity in $c_{i}$ 's.

- Given $c_{i}=c$ and $\delta_{i}=\delta$ for all $i \in N$, SC increases with $n$.

To understand Proposition 3, I first note that all else being equal, an agent's equilibrium payoff increases at an increasing rate in his discount factor. ${ }^{16}$ This is because a higher discount factor improves one's bargaining power not only in the current period but also in every period following a rejection, all of which then feed back into the equilibrium payoff. Armed with this observation, the intuition behind the first part of Proposition 3 easily follows: As the group becomes more heterogenous in time preferences, it contains agents with discount factors closer to both extremes. Although the presence of less patient agents who, according to Proposition 1, demand a lower share from the surplus raises the prize from proposing, the presence of more patient agents reduces it, to the extent that the overall prize, i.e., $\pi_{i}^{n}$, is smaller, and so is the incentive to propose. Put differently, the presence of "tough" bargainers who refuse to settle for little helps reduce wasteful efforts. ${ }^{17}$ Indeed, as some agent $i$ becomes arbitrarily patient, the social cost vanishes, i.e., $S C \rightarrow 0$, for $\delta_{i} \rightarrow 1$. However, it is also the agent $i$ in this case who benefits most from the cost savings, since $v_{i} \rightarrow 1$ for $\delta_{i} \rightarrow 1$. A similar intuition holds for cost heterogeneity. ${ }^{18}$ In particular, a lower marginal cost raises one's payoff not only because of its direct effect but also because of its strategic effect. Finally, the competition becomes more intense in a larger group, as

\footnotetext{
${ }^{15}$ In general, $\theta^{\prime}=\left(\theta_{1}^{\prime}, \ldots, \theta_{n}^{\prime}\right)$ is a mean-preserving spread of $\theta^{\prime \prime}=\left(\theta_{1}^{\prime \prime}, \ldots, \theta_{n}^{\prime \prime}\right)$ if (1) $\sum_{i} \theta_{i}^{\prime}=\sum_{i} \theta_{i}^{\prime \prime}$ and (2) $\theta_{j}^{\prime} \geqslant \theta_{j}^{\prime \prime}$ if and only if $\theta_{j}^{\prime \prime} \geqslant \frac{1}{n} \sum_{i} \theta_{i}^{\prime \prime}$ for $j \in N$.

16 This property can be easily verified in general, and it also holds for the standard two-agent Rubinstein bargaining.

${ }^{17}$ The rent-seeking literature also recognizes the fact that unevenly matched contenders might waste fewer resources. See, e.g., Che and Gale [9], Esteban and Ray [12], Leininger [20], and Nti [26]. Viewed as a one-shot rent-seeking game in equilibrium, agents in the present bargaining setup are identical with respect to the prize they compete for. The heterogeneity comes into play in determining the endogenous prize.

${ }^{18}$ Although the analysis of cost heterogeneity requires an additional restriction that $f\left(x_{i}\right)=x_{i}$, I conjecture it will hold in general. Yet, I have been unable to prove this.
} 
recorded in the last part of Proposition 3. The reason is that as the number of negotiating agents increases, they expect recognition to be costlier and therefore have lower continuation values. This raises the prize from proposing by making votes cheaper to buy out ${ }^{19}$ and consequently heats up the competition to propose.

The role of group heterogeneity in reducing the social cost can provide an alternative explanation as to why organizations may treat identical agents unequally. For instance, professional partnerships and academic departments assign titles such as associate and senior associate to their members, which make some members more permanent in the organization than others. These titles often are not linked to significant job differences, and as the model predicts, more permanent members receive a greater share from the surplus. ${ }^{20}$

\section{4. k-Majority rule}

Inow relax the unanimity rule assumption granting each agent veto power, and extend the framework to general voting procedures in which a proposal requires $k \in\{1, \ldots, n\}$ votes (including the proposer's) to be accepted. The analysis, however, becomes significantly more complicated. This is because a proposer wants to buy out the votes of the cheapest "winning coalition", which may vary across agents.

Let $\psi_{i j}$ be the probability that player $i$ includes $j$ in his offer or winning coalition. We modify (1) as follows. If player $i$ is recognized with $p_{i}(\mathbf{x})$, then he pays the continuation values of players in his winning coalition. Let $w_{i}$ denote the total payment made by player $i$, where

$$
w_{i} \equiv \sum_{j \neq i} \psi_{i j} \delta_{j} v_{j}
$$

If player $i$ is not recognized, however, he will be offered his continuation value, as long as he is in the proposer's winning coalition, which happens with probability $\mu_{i}(\mathbf{x})$, where

$$
\mu_{i}(\mathbf{x}) \equiv \sum_{j \neq i} p_{j}(\mathbf{x}) \psi_{j i}
$$

and $0 \leqslant \mu_{i}(\mathbf{x}) \leqslant 1-p_{i}(\mathbf{x})$.

Overall, player $i$ solves the following dynamic program:

$$
v_{i}=\max _{x_{i} \in\left[0, \frac{1}{c_{i}}\right]}\left\{p_{i}(\mathbf{x})\left(1-w_{i}\right)+\mu_{i}(\mathbf{x}) \delta_{i} v_{i}-c_{i} x_{i}\right\} .
$$

The optimal effort choice requires ${ }^{21,22}$

$$
\text { FOC }: \frac{\partial p_{i}(\mathbf{x})}{\partial x_{i}} \pi_{i}^{k}-c_{i} \leqslant 0 \quad\left(=0 \text { if } x_{i}>0\right)
$$

\footnotetext{
${ }^{19}$ Indeed, given $\delta_{i}=\delta$ for all $i, \pi_{i}^{n}=1-n \delta v_{i}$ increases in $n$.

${ }^{20}$ In a recent paper, Winter [38] provides an alternative incentive-based explanation for the same phenomenon. He argues that identical agents each performing a complementary task may be rewarded differently for a successful project to minimize the coordination problem among them.

${ }^{21}$ To save on notation, I sometimes omit arguments of functions whenever there is no confusion.

${ }^{22}$ Given that $\frac{\mu_{i}(\mathbf{x})}{1-p_{i}(\mathbf{x})}=\sum_{j \neq i} \frac{f\left(x_{j}\right)}{\sum_{j \neq i} f\left(x_{j}\right)} \psi_{j i}$, which is independent of $x_{i}$, it is clear that the SOC holds.
} 
where the prize from proposing is now $\pi_{i}^{k} \equiv 1-w_{i}-\frac{\mu_{i}}{1-p_{i}} \delta_{i} v_{i}$ and I make use of the facts $\frac{\partial p_{i}}{\partial x_{i}}=\frac{\varepsilon\left(x_{i}\right)}{x_{i}} p_{i}\left(1-p_{i}\right)$ and $\frac{\partial p_{j}}{\partial x_{i}}=-\frac{\varepsilon\left(x_{i}\right)}{x_{i}} p_{i} p_{j}$ for $j \neq i$.

Eq. (11) reveals that as for the unanimity rule, player $i$ 's effort choice is shaped by the prize and cost effects. The prize from proposing, $\pi_{i}^{k}$, is once again the difference between the residual surplus, $1-w_{i}$, to be gained from being the proposer and the forgone expected payment, $\frac{\mu_{i}}{1-p_{i}} \delta_{i} v_{i}$, to be earned from being a nonproposer but included in the winning coalition. ${ }^{23}$ For the unanimity rule, since $\mu_{i}=1-p_{i}, \pi_{i}^{k}$ reduces to $\pi_{i}^{n}$, as it should. Unlike under the unanimity rule, however, agents do not necessarily compete for the same prize under a $k$-majority rule, because each proposer may include a different set of $k-1$ players in the proposal. Nonetheless, it seems intuitive that agents with relatively more expensive votes would expect to be excluded from winning coalitions and thus would have more to gain from proposing. The following result confirms this intuition and provides an additional insight.

Lemma 2. Suppose the voting rule is k-majority and $p_{i}(\mathbf{x})$ satisfies A.2. Moreover, suppose, in equilibrium, one of the following two conditions holds: for some $i, j \in N$,

- $\delta_{j} v_{j}<\delta_{i} v_{i}$, or

- $\delta_{j} v_{j}=\delta_{i} v_{i}, \delta_{j} \leqslant \delta_{i}$ and $c_{i} \leqslant c_{j}$.

Then, in equilibrium, $\pi_{j}^{k} \leqslant \pi_{i}^{k}$.

Recall that when not the proposer, player $i$ 's vote can be bought out by paying him his continuation payoff, $\delta_{i} v_{i}$. Thus, the first part of Lemma 2 provides a convenient comparison of the prizes based on the "prices" of agents' votes. The second part reveals that even if the equilibrium prices of two agents' votes are equal, they might expect different prizes from proposing. This is because the more patient and/or more efficient agent is supposed to have a greater off-equilibrium continuation value, and thus he is more likely to be excluded from winning coalitions. This raises his stakes from proposing, and induces him to incur a greater effort cost, which, in turn, lowers his off-equilibrium continuation value. (This point is illustrated in Example 2 below.) The following proposition further refines our intuition.

Proposition 4. Suppose $p_{i}(\mathbf{x})$ satisfies A.2. Then, under a k-majority voting rule, there exists a unique equilibrium pair of $(\mathbf{x}, \mathbf{v})$ and it has these properties: $x_{l}>0$ for some $l \in N$, and if $\delta_{j} \leqslant \delta_{i}$ and $c_{i} \leqslant c_{j}$ for some $i, j \in N$, then

- $\delta_{i} v_{i} \geqslant \delta_{j} v_{j}$.

- $x_{i} \geqslant x_{j}$.

- $p_{i}(\mathbf{x}) \geqslant p_{j}(\mathbf{x})$.

According to the first part of Proposition 4, a more patient and/or more efficient agent has a higher continuation value. This is because if in the winning coalition, such an agent can generate a favorable offer for himself by simply rejecting the unfavorable ones. However, this also means his vote is relatively more expensive, and thus less desirable to be bought out. Realizing this possibility, such an agent attaches a greater prize to proposing (implied by Lemma 2 ) and hence exerts a greater

\footnotetext{
${ }^{23}$ Indeed, as alluded to earlier, in equilibrium, the bargaining game reduces to a one-shot rent-seeking game where player $i$ wins the prize $\pi_{i}^{k}$. Note also that $\frac{\mu_{i}}{1-p_{i}}$ is the probability that player $i$ is in the winning coalition conditional on not proposing.
} 
Table 1

Dissipation of cost advantage

\begin{tabular}{lccc}
\hline & Agent 1 & Agent 2 & Agent 3 \\
\hline$c_{i}$ & 1 & 1.02 & 1.05 \\
$v_{i}$ & .159 & .159 & .159 \\
$p_{i}$ & .364 & .337 & .299 \\
$\pi_{i}$ & .804 & .787 & .766 \\
$\mu_{i}$ & .234 & .322 & .443 \\
\hline
\end{tabular}

effort to propose, as recorded in the rest of the proposition. In terms of the equilibrium, there is a trivial multiplicity (see also [2,3]). ${ }^{24}$ Yet, the equilibrium effort and payoffs are unique, which serves our purposes. 25

While Proposition 4 provides a clear and intuitive comparison of agents' equilibrium continuation values, it is also important to compare their expected payoffs, $v_{i}$. Observe that if $\delta_{i}=\delta_{j}$ for agents $i$ and $j$, then Proposition 4 implies that the more efficient agent obtains a weakly higher payoff. Under the unanimity rule, this inequality becomes strict whenever the agents in question exert positive efforts (Proposition 2). However, it turns out that the same is not true for a nonunanimity rule. That is, even if $\delta_{i}=\delta_{j}$ and $c_{i}<c_{j}$ for some $i, j \in N$, it is possible that $v_{i}=v_{j}$. I illustrate this point and the role of cost heterogeneity on the social cost in the following example, and then turn my attention to the case where agents have equal costs but different discount factors in Example 3.

Example 2. Consider a three-agent bargaining, where agreement requires a simple majority, i.e., $n=3$ and $k=2$. Moreover, let $c_{1} \leqslant c_{2} \leqslant c_{3}, \delta_{i}=\delta$ for all $i$, and $p_{i}(\mathbf{x})$ satisfy A.2 with $f\left(x_{i}\right)=x_{i}$.

Dissipation of cost advantage: One can show that all agents obtain the same equilibrium payoff given by

$$
v_{i}=\frac{\sum_{i} \frac{1}{1+p_{i}}-2}{(1+\delta) \sum_{i} \frac{1}{1+p_{i}}-3 \delta}
$$

if and only if $c_{1}\left(c_{2}+c_{3}\right)-c_{2} c_{3} \geqslant 0$. That is, an equal-payoff equilibrium exists if only if agents' marginal costs are not too different. To better explain the intuition, I consider the following numerical example with $\delta=.9$ summarized in Table 1. Inspecting Table 1, note, for instance, that agent 1 , being the most efficient, is expected to propose with the highest probability and have the highest (off-equilibrium) continuation payoff, which makes his vote the least desirable by others, as reflected in $\mu_{i}$ 's. This in turn makes his prize from proposing the highest, and leads him to exert the highest effort, dissipating his initial cost advantage. As noted above, there is also a trivial multiplicity of equilibria here. In particular, agents form their coalitions with these mixed strategies: $\psi_{12}=.821\left(.078+\psi_{31}\right)$ and $\psi_{21}=.886\left(.782-\psi_{31}\right)$ for $\psi_{31} \in[0, .782]$.

\footnotetext{
${ }^{24}$ Example 2 demonstrates this trivial multiplicity of equilibria.

${ }^{25}$ Eraslan [10] shows the uniqueness of equilibrium payoffs in the Baron and Ferejohn model with an exogenous recognition rule.
} 
Table 2

\begin{tabular}{llcc}
\hline & Agent 1 & Agent 2 & Agent 3 \\
\hline (a) & .8 & & 1.2 \\
$c_{i}$ & .223 & 1 & .136 \\
$v_{i}$ & .504 & .137 & .135 \\
$p_{i}$ & .877 & .361 & .753 \\
$\pi_{i}$ & 0 & .851 & .865 \\
$\mu_{i}$ & & .135 & \\
$(\mathrm{~b})$ & .7 & & .3 \\
$c_{i}$ & .307 & 1 & .037 \\
$v_{i}$ & .564 & .143 & .067 \\
$p_{i}$ & .966 & .369 & .837 \\
$\pi_{i}$ & 0 & .952 & .933 \\
$\mu_{i}$ & 0 & .067 & \\
\hline
\end{tabular}

The possibility of an increase in social cost with cost heterogeneity: Let us first examine the impact of cost heterogeneity on the social cost when, in equilibrium, agents end up with equal payoffs. Since $\frac{1}{1+p_{i}}$ is decreasing and convex in $p_{i}$, the payoff $v_{i}$ in (12) increases in cost heterogeneity, leading to a lower social cost $\sum_{i} c_{i} x_{i}=1-\sum_{i} v_{i}$. This similarity to the case with unanimity rule (Proposition 3 ) comes from the fact that all agents have a positive and significant probability of being included in winning coalitions, which curbs their incentives to propose. However, unlike the unanimity rule, when cost heterogeneity is so severe that low-cost agents are excluded in the others' offers, this conclusion may be reversed with the majority rule. I now demonstrate this point by allowing agents' marginal costs to be sufficiently different so that in equilibrium $v_{3}<v_{2}<v_{1}$. In terms of cost heterogeneity, I consider the following meanpreserving spread on marginal costs: $c_{1}+c_{3}=2$ and $c_{2}=1$. The equilibrium outcomes for two cases are reported in Table $2 \mathrm{a}$ and $\mathrm{b}$.

Comparing Table $2 \mathrm{a}$ and $\mathrm{b}$, note that the social cost, $\sum_{i} c_{i} x_{i}=1-\sum_{i} v_{i}$, goes up from .504 to .513 , as the group becomes more heterogenous. The intuition is that a more heterogenous group in Table $2 \mathrm{~b}$ contains a more efficient agent 1 and a less efficient agent 3 . While this means a greater payoff for 1 and a lower payoff for 3, contrary to the case with the unanimity rule, it increases the stakes from proposing for all agents. This is because the most expensive vote of agent 1 is not needed by others.

Next, I turn my attention to the impact of patience on the equilibrium outcome with majority rule. To do so, I assume that agents possess equal marginal costs but different discount factors. Under the unanimity rule, Proposition 1 implies that the more patient agent necessarily receives a greater payoff. Under a nonunanimity rule, however, this is not true. Furthermore, unlike the case with heterogenous costs, although the more patient agent obtains a weakly higher continuation value, he may end up with a lower payoff than a less patient agent. I demonstrate these points by continuing the setup in Example 2.

Example 3. Consider again a three-agent bargaining, where agreement requires a simple majority, and $p_{i}(\mathbf{x})$ satisfies A.2 with $f\left(x_{i}\right)=x_{i}$. Also, let $c_{i}=1$ for all $i$. Suppose the equilibrium continuation values are such that $\delta_{1} v_{1}<\delta_{2} v_{2}<\delta_{3} v_{3}$. If recognized in a given period, 
agent 1 then offers an allocation $\left(s_{1}, s_{2}, s_{3}\right)=\left(1-\delta_{2} v_{2}, \delta_{2} v_{2}, 0\right)$ whereas agents 2 and 3 offer $\left(\delta_{1} v_{1}, 1-\delta_{1} v_{1}, 0\right)$ and $\left(\delta_{1} v_{1}, 0,1-\delta_{1} v_{1}\right)$, respectively. These allocations imply (a) $w_{1}=\delta_{2} v_{2}$, $w_{2}=w_{3}=\delta_{1} v_{1}$, and (b) $\mu_{1}=1-p_{1}, \mu_{2}=p_{1}, \mu_{3}=0$.

Disadvantages of being patient: It can be verified that in equilibrium, recognition probabilities are given by

$$
p_{1}=\frac{1}{3}\left(2-\frac{\pi_{3}}{\pi_{1}}\right), \quad p_{2}=\frac{1}{3}\left(\frac{\pi_{3}}{\pi_{1}}+\frac{\pi_{1}}{\pi_{3}}-1\right), \quad \text { and } \quad p_{3}=\frac{1}{3}\left(2-\frac{\pi_{1}}{\pi_{3}}\right) .
$$

Moreover, the following equation uniquely identifies $\frac{\pi_{1}}{\pi_{3}}$ for a given $\delta_{2}$ :

$$
1-\frac{\pi_{1}}{\pi_{3}}=\frac{\delta_{2} p_{2}^{2}}{1-\delta_{2} p_{1}\left(1+p_{2}\right)} .
$$

Since there is no convenient closed-form solution to (14), however, I proceed by fixing $\delta_{2} \cdot{ }^{26}$ Let $\delta_{2}=.6$. Then, (14) reveals $\frac{\pi_{1}}{\pi_{3}}=.911$, which in turn reveals $p_{1}=.301, p_{2}=.336$, and $p_{3}=.363$. Moreover, from (10) and (11), we have $\frac{v_{1}}{v_{2}}=\frac{0.554}{1-\delta_{1}}$ and $\frac{v_{2}}{v_{3}}=1.131$. The initial equilibrium condition $\delta_{1} v_{1}<\delta_{2} v_{2}<\delta_{3} v_{3}$ we imposed is now satisfied if $\delta_{1} \in[0, .519)$ and $\delta_{3} \in[.678,1)$. With these restrictions in mind, we find $\frac{v_{1}}{v_{2}}>1$ if and only if $\delta_{1} \geqslant .446$. In sum, for $\delta_{1} \in[0, .519], \delta_{2}=.6$ and $\delta_{3} \in[.678,1)$, we have $\delta_{1} v_{1}<\delta_{2} v_{2}<\delta_{3} v_{3}$ and

$$
\begin{aligned}
& v_{1} \geqslant v_{2}>v_{3} \quad \text { if } \delta_{1} \in[.446, .519), \\
& v_{2}>v_{1} \geqslant v_{3} \quad \text { if } \delta_{1} \in[.373, .446), \\
& v_{2}>v_{3}>v_{1} \quad \text { if } \delta_{1} \in[0, .373) .
\end{aligned}
$$

As a benchmark, I also consider the case where recognition is costless, and $p_{i}=\frac{1}{3}$ for all $i$. For $\delta_{2}=.6, \delta_{1} \in[0, .5)$ and $\delta_{3} \in[.75,1)$, we have the equilibrium with $\delta_{1} \widetilde{v}_{1}<\delta_{2} \widetilde{v}_{2}<\delta_{3} \widetilde{v}_{3}$, and

$$
\begin{array}{ll}
\widetilde{v}_{1} \geqslant \widetilde{v}_{2}>\widetilde{v}_{3} & \text { if } \delta_{1} \in[.4, .5), \\
\widetilde{v}_{2}>\widetilde{v}_{1} \geqslant \widetilde{v}_{3} & \text { if } \delta_{1} \in[.25, .4), \\
\tilde{v}_{2}>\widetilde{v}_{3}>\widetilde{v}_{1} & \text { if } \delta_{1} \in[0, .25) .
\end{array}
$$

Two insights emerge from the discussion so far. First, the impact of patience level on equilibrium payoffs changes dramatically with the voting rule. In particular, the agent who is more patient does not necessarily receive a higher payoff, when agreement requires a less than unanimous approval. In fact, for certain values of discount factors, the lowest payoff accrues to the most patient agent, i.e., agent $3 .^{27}$ The intuition is that by being patient, agent 3 is expected to possess a greater continuation payoff, making his vote more expensive. With the majority voting rule, however, this means agent 3 is excluded from the offers, when he is not the proposer. Second, comparing (15) and (16), we see that when agents can influence their recognition, a more patient agent is

\footnotetext{
${ }^{26}$ After some simplification, (14) reduces to $\delta_{2}\left(\frac{\pi_{1}}{\pi_{3}}\right)^{3}-3\left(3-\delta_{2}\right)\left(\frac{\pi_{1}}{\pi_{3}}\right)^{2}+3\left(3-2 \delta_{2}\right)\left(\frac{\pi_{1}}{\pi_{3}}\right)+\delta_{2}=0$.

27 This observation might seem puzzling in light of Corollary 2 of Eraslan [10], in which she notes, given the exogenous recognition rule $p_{i}=\frac{1}{n}$ and under general voting rules, patient agents cannot be worse off. I am grateful to Hulya Eraslan for pointing out an error in her finding.
} 
able to tip the proposal power in his favor, and reduce the disadvantage of being excluded from the offers. For instance, for $\delta_{1} \in[.25, .373], \delta_{2}=.6$ and $\delta_{3} \in[.75,1)$, whereas $\widetilde{v}_{1} \geqslant \widetilde{v}_{3}$, we have $v_{3}>v_{1}$.

The possibility of an increase in social cost with discount factor heterogeneity: Let us make the following mean-preserving spread on discount factors: $\delta_{2}=.6$ and $\delta_{1}+\delta_{3}=1$, where $\delta_{1} \in[0, .519]$ and $\delta_{3} \in[.678,1)$. Clearly, as $\delta_{1}$ decreases and $\delta_{3}$ increases, the group becomes more heterogenous. From (11), the social cost is given by $S C=\sum_{i} x_{i}=\sum_{i} p_{i}\left(1-p_{i}\right) \pi_{i}$. Eq. (14) reveals that $\frac{\pi_{1}}{\pi_{3}}$ depends only on $\delta_{2}$, and from (13), so do $p_{i}$ 's. Moreover, using the definition of $\pi_{i}$ 's, we obtain

$$
\begin{aligned}
\pi_{1} & =\frac{1-\zeta}{1+\eta(1-\zeta)}, \\
\pi_{3} & =\frac{1}{1+\eta(1-\zeta)}, \\
\pi_{2} & =\frac{p_{1}}{p_{1}+p_{3}} \pi_{1}+\frac{p_{3}}{p_{1}+p_{3}} \pi_{3},
\end{aligned}
$$

where $\zeta \equiv \frac{\delta_{2} p_{2}^{2}}{1-\delta_{2} p_{1}\left(1+p_{2}\right)}$ and $\eta \equiv \frac{\delta_{1}}{1-\delta_{1}} p_{1}^{2}$.

Note that as the group becomes more heterogenous, all $\pi_{i}$ 's and hence the social cost increase. This is in sharp contrast with the finding under the unanimity rule (Proposition 2) that heterogeneity in discounting always lowers social cost. The reason is twofold. First, unlike the unanimity rule, the presence of a more patient agent 3 has no cost-reducing effect, since his expensive vote need not be bought out under the majority rule. Second, as agent 1 becomes less patient, his vote gets cheaper, which raises the prize from proposing and intensifies the competition to propose.

\section{Voting rules and distribution of surplus}

Up to now, my analysis has focused on agents' equilibrium payoffs net of recognition costs. Perhaps equally important is the distribution of surplus, and it is well-known that the distribution is skewed toward the proposer's favor, as he gains a (temporary) monopoly power over nonproposers. ${ }^{28}$ The extent of this power, however, hinges critically on the nonproposers' ability to reject an offer and wait for future ones. To investigate various factors that affect the equilibrium distribution of surplus, I restrict the previous analysis and assume that agents are identical, i.e., $c_{i}=c$ and $\delta_{i}=\delta$ for all $i \in N$. Thus, there is a unique equilibrium pair of $(\mathbf{x}, \mathbf{v})$ such that all agents (a) possess the same payoff, $v$, (b) exert the same effort, $x$, and (c) propose with equal probability, i.e., $p_{i}=\frac{1}{n}$. In what follows, it is also more convenient to represent voting rules as the " $r$-majority", by defining $k \equiv r n+(1-r)$ for some $r \in[0,1] .{ }^{29}$ For instance, the unanimity and the simple majority rules are equivalent to $r=1$ and $\frac{1}{2}$, respectively. Since each player is equally likely to be in a winning coalition when not the proposer, $\psi_{i j}=\frac{k-1}{n-1}=r$ and $\mu_{i}=\frac{k-1}{n}=\frac{r(n-1)}{n}$.

\footnotetext{
${ }^{28}$ An obvious source of this monopoly power is nonproposers' discounting of the future. For nonunanimity voting rules, even if they do not discount the future, nonproposers may still value the future less, because of the fear of not being included in the winning coalition.

${ }^{29}$ In particular, the comparative static with respect to $n$ becomes obscure with the $k$-majority, because $k$ itself is often a function of $n$.
} 
Inserting these facts into (10) yields

$$
v=\frac{1}{n}-c x \text {. }
$$

From (17), it is clear that in the absence of costly recognition, i.e., $x \rightarrow 0$, each agent receives an ex ante payoff of $v=\frac{1}{n}$, regardless of the voting rule and agents' patience [2]. The reason is that without costly recognition, there are no equilibrium inefficiencies. The following result summarizes how $x$ (and hence $v$ in (17)) changes with various parameters.

Proposition 5. Suppose that $c_{i}=c$ and $\delta_{i}=\delta$ for all $i \in N$, and that $p_{i}(\mathbf{x})$ satisfies A.2. Then, there is a unique and symmetric equilibrium pair of $(\mathbf{x}, \mathbf{v})$ such that $v$ increases in $r$ and $\delta$, and decreases in $n$ whereas $x$ decreases in $r$ and $\delta$, and $n c x$ increases in $n$.

When recognition is costly, Proposition 5 reveals that $v$ increases and $x$ decreases in $r$. As agreement requires more affirmative votes, it improves not only social efficiency but, interestingly, the individual payoff as well. The intuition is that whereas an increase in $r$ reduces the payoff by requiring the proposer to buy out more votes, it also collectively and credibly commits agents not to exert too much effort by lowering the prize from proposing. ${ }^{30}$ As a third effect, an increase in $r$ also makes it more likely for a nonproposer to be included in an offer. Overall, the two positive effects associated with an increase in $r$ outweigh the negative effect, and hence the individual payoff goes up. Similarly, when agents are more patient, their expected payoffs and hence the continuation values increase, making their votes more expensive. This in turn reduces the prize from proposing and the effort to propose, benefiting all agents. The last part of Proposition 5 reveals that as $n$ increases, given more intense competition, so does the total cost, $n c x$. This, however, reduces the individual payoff, $v$, as expected. ${ }^{31}$

In equilibrium, the surplus is divided between agents, in particular, between the proposer and nonproposers. The proposer receives a share of $s_{p} \equiv 1-(k-1) \delta v=1-\delta r(n-1) v$, whereas each nonproposer expects to receive $s_{n p} \equiv \frac{k-1}{n-1} \delta v=\delta r v$. Thus, the expected gain from proposing is

$$
\Delta \equiv s_{p}-s_{n p}=1-\delta r n v .
$$

Corollary 1. $\Delta$ decreases in $\delta$ and $r$, and increases in $n$.

To understand Corollary 1 , note first that for $x \rightarrow 0$, Eq. (18) reduces to $\Delta_{0}=1-\delta r$. That is, in the absence of costly recognition, since nonproposers' payoffs are proportional to surplus, the expected gain from proposing is independent of $n$. Furthermore, since nonproposers possess a greater continuation payoff when they are more patient and/or a decision requires more unanimous agreement, $\Delta_{0}$ is decreasing in $\delta$ and $r .{ }^{32}$ In the presence of costly recognition, how much nonproposers demand also depends on the degree of competition to propose. In particular, any factor

\footnotetext{
${ }^{30}$ It is easy to verify that the equilibrium prize from proposing is $\pi_{i}^{r}=1-\delta r n v$ and given that $v$ increases in $r$, it decreases in $r$.

${ }^{31}$ It is interesting to note that the effect of $n$ on $x$ is, in general, ambiguous (see Proof of Proposition 5). This is because as $n$ increases, while each agent expects to propose with a lower probability, he also expects to receive a greater prize from proposing. The latter force is absent when $\delta=0$ or $r=0$, in which case the competition reduces to a one-shot rent-seeking game.

32 These observations are consistent with Harrington [16], who also considers the equilibrium distribution of surplus in a symmetric model much like Baron and Ferejohn [1,2] but with risk-averse players.
} 
that intensifies the competition lowers future (off-equilibrium) payoffs, and therefore provides the proposer with an additional monopoly power over nonproposers. As with the unanimity case, an increase in $n$ and/or a decrease in $\delta$ result in a greater competition, as recorded in Proposition 5. Similarly, as agreement requires more affirmative votes, the expected continuation payoff goes up for nonproposers, which reduces the proposer's expected gain.

Before proceeding, it is interesting to relate our results in this section to those of Inderst et al. [18]. As does the present research but within a static framework, Inderst et al. take a political view of resource allocation within organizations, where agents exert rent-seeking efforts to grab a share. They show that multidivisional organizations may manage wasteful activities more effectively than do single-tier ones, even though they offer more scope for organizational conflict-both at inter and intra-divisional levels. The main reason is that intra-divisional rent-seeking takes place only for a fraction of the overall prize. In the same vein, Muller and Warneryd [24] argue that in the absence of complete contracts, managers of a firm might prefer outside ownership to reduce internal conflict between them by essentially committing to lowering the prize to fight for. Combining these insights with my findings reveals the following testable observations: Organizations and firms that require more consensus in resource allocation are (a) less likely to divisionalize, and (b) less likely to benefit from outside ownership. ${ }^{33}$

\section{An extension: costly recognition with persistence}

The analysis thus far has assumed that the recognition probabilities depend only on the current efforts. This seems a good approximation in cases where agents need to "push" for their proposals repeatedly. For instance, nations disputing over a territory might have to generate repeated support from the same countries with frequently changing leadership. Nonetheless, in many other cases, recognition appears to have a persistent or intrinsic component as well. For instance, negotiating agents might have already established some recognition on a specific issue, but their current efforts play a role, too. To capture this possibility, I now assume agent $i$ is recognized with probability $q_{i}(\mathbf{x})$ that satisfies ${ }^{34}:$

A.3. $q_{i}(\mathbf{x})=\lambda \alpha_{i}+(1-\lambda) p_{i}(\mathbf{x})$, where $\lambda \in[0,1], \alpha_{i} \geqslant 0$ for all $i \in N$, and $\sum_{i} \alpha_{i}=\sum_{i} p_{i}(\mathbf{x})=1$

for all $\mathbf{x}$.

The bargaining model with $q_{i}(\mathbf{x})$ literally combines the one with exogenous probabilities, $\alpha_{i}$, and the one examined above, admitting each as a special case. Thus, I only examine cases with $\lambda \in(0,1)$ here. To see the potential impact of this generalization, consider first the case with the unanimity rule. By replacing $p_{i}(\mathbf{x})$ with $q_{i}(\mathbf{x})$ in (2), we obtain

$$
v_{i}=\max _{x_{i} \in\left[0, \frac{1}{c_{i}}\right]}\left\{q_{i}(\mathbf{x})\left[1-\sum_{j} \delta_{j} v_{j}\right]+\delta_{i} v_{i}-c_{i} x_{i}\right\} .
$$

Differentiating the r.h.s. with respect to $x_{i}$ and defining $\widehat{c}_{i} \equiv \frac{c_{i}}{1-\lambda}$ yield

$$
\text { FOC }: \frac{\partial p_{i}(\mathbf{x})}{\partial x_{i}}\left[1-\sum_{j} \delta_{j} v_{j}\right]-\widehat{c}_{i} \leqslant 0 \quad\left(=0 \text { if } x_{i}>0\right) .
$$

\footnotetext{
${ }^{33}$ Note that the voting procedure has no bite in the static models of Inderst et al., and Muller and Warneryd.

${ }^{34}$ I am grateful to a referee for suggesting this extension.
} 
Since (20) coincides with (3), it is clear that Proposition 1 holds with only a trivial modification. In particular, for $c_{i}=c$ for all $i$, we once again have $x_{i}=x_{j}$ and $p_{i}=p_{j}=\frac{1}{n}$. This implies, in equilibrium, that $q_{i}=\lambda \alpha_{i}+(1-\lambda) \frac{1}{n}$ and that if $\alpha_{i}>\alpha_{j}$, then $q_{i} \geqslant q_{j}$ and $\left(1-\delta_{i}\right) v_{i} \geqslant\left(1-\delta_{j}\right) v_{j}$. When agents also differ in their marginal costs, Proposition 2 follows with a slight modification, too. For instance, if $c_{i}<c_{j}$, then (20) reveals that $x_{i} \geqslant x_{j}$ and thus $p_{i} \geqslant p_{j}$. One then needs to compare $q_{i}$ and $q_{j}$ using the information on $\alpha_{i}$ and $\alpha_{j}$.

When the voting rule is less than unanimity, it turns out that the impact of the persistence in recognition is similar to that of the marginal cost. To see this, let agents differ only in their $\alpha_{i}$ 's. Intuitively, the agent with a higher $\alpha_{i}$ is expected to propose with a greater probability, and possess a greater expected net payoff. This, however, makes his vote more expensive for others to buy out and gives him an additional incentive to expend effort to propose. I confirm this intuition in the following result.

Proposition 6. Suppose that $\delta_{i}=\delta$ and $c_{i}=c$ for all $i$ and that $p_{i}(\mathbf{x})$ and $q_{i}(\mathbf{x})$ satisfy A.2 and A.3 with $\lambda \in(0,1)$, respectively. Then, there exists a unique equilibrium pair of $(\mathbf{x}, \mathbf{v})$ such that if $\alpha_{i}>\alpha_{j}$, then

- $x_{i} \geqslant x_{j}$.

- $q_{i}(\mathbf{x})>q_{j}(\mathbf{x})$.

- $v_{i} \geqslant v_{j}$.

The discussion so far suggests that the extension using A.3 does not alter our previous results with $\lambda=0$ in any qualitative way. As expected, all else equal, the agent with a greater intrinsic recognition proposes with a greater probability and is weakly better off. Perhaps, an interesting new insight is that, all else being equal, the agent with a greater intrinsic recognition tends to reinforce his proposal power by exerting a greater effort.

Related to this extension, one can also imagine that the intrinsic component itself is determined by the current and past efforts. That is, agent $i$ 's intrinsic recognition in period $t \in\{0,1, \ldots\}$ is given by $\alpha_{i, t}=\varphi_{i}\left(\mathbf{X}_{1, t}, \ldots, \mathbf{X}_{n, t}\right)$, where $\mathbf{X}_{i, t}=\left(x_{i, 0}, x_{i, 1}, \ldots, x_{i, t-1}\right)$ denotes $i$ 's effort history with $x_{i,-1}=0$. While a full development of this generalization is beyond the scope of this paper, the following example demonstrates the value of future research in this direction.

Example 4. Consider the two-agent setup in Example 1, but now suppose agents simultaneously choose their efforts once-and-for-all in period 0 . These efforts are then observed by both agents, and they determine agents' recognition probabilities in the subsequent bargaining game. In the unique equilibrium, both agents choose strictly positive efforts, and the total cost for agent $i$ is given by

$$
c_{i} \widehat{x}_{i}=\frac{\left(1-\delta_{i}\right)\left(1-\delta_{j}\right)}{\frac{c_{i}}{c_{j}}\left(1-\delta_{i}\right)^{2}+\frac{c_{j}}{c_{i}}\left(1-\delta_{j}\right)^{2}+2\left(1-\delta_{i}\right)\left(1-\delta_{j}\right)} .
$$

This means that as in Example 1, agent $i$ proposes with $p_{i}=\frac{c_{j}}{c_{i}+c_{j}}$. His expected share from the surplus is

$$
\widehat{s}_{i}=\frac{1-\delta_{j}}{\left(1-\delta_{j}\right)+\frac{c_{i}}{c_{j}}\left(1-\delta_{i}\right)} .
$$


Subtracting (21) from (22), we compute $i$ 's expected payoff:

$$
\widehat{v}_{i}=\frac{\left(1-\delta_{j}\right)^{2}}{\left(1-\delta_{j}\right)^{2}+\left(\frac{c_{i}}{c_{j}}\right)^{2}\left(1-\delta_{i}\right)^{2}+2 \frac{c_{i}}{c_{j}}\left(1-\delta_{i}\right)\left(1-\delta_{j}\right)} .
$$

It is clear that the qualitative properties of $c_{i} \widehat{x}_{i}, \widehat{s}_{i}$, and $\widehat{v}_{i}$ are the same as in Example 1. However, comparing the equilibrium outcomes across two examples yields insights into the role of persistence. First, although agents end up proposing with equal probabilities in both cases, they do exert greater efforts when efforts have a persistent effect on recognition. Formally, $\widehat{x}_{i} \geqslant x_{i}$ with strict inequality when $\delta_{i} \neq 0$. The reason is that knowing that the current effort will determine the recognition throughout the bargaining, agents expect a higher marginal return on their investments. Second, from (5) and (22), it follows that $\widehat{s}_{i}>\bar{s}_{i}$ if and only if $c_{i} \delta_{i}>c_{j} \delta_{j}$. That is, the more efficient and/or more patient agent grabs a larger share from the surplus when efforts are persistent, making the allocation more unequal. The intuition is that when efforts are transitory, agents know that after every rejection, they will waste resources to be recognized. To avoid this possibility, the advantageous agent is willing to make a more generous offer to the other, which leads to a more equal allocation. Finally, comparing (6) and (23), we see that $v_{i} \geqslant \widehat{v}_{i}$ if and only if $2 \delta_{j}\left(1-\delta_{j}\right)+\frac{c_{i}}{c_{j}}\left(\delta_{j}-\delta_{i}\right) \geqslant 0$. This condition clearly holds if $\delta_{j} \geqslant \delta_{i}$. That is, the less patient agent strictly prefers the case with the transitory effect. This makes sense. In such a case, the less patient agent not only exerts less effort, but he also obtains a more generous offer. However, whether the more patient agent is better off with the case of persistence is not clear. For instance, if $\delta_{j}<\delta_{i}$ and $\delta_{j} \approx \delta_{i}$, it is true that $v_{i}>\widehat{v}_{i}$. On the other hand, if $\delta_{j}<\delta_{i}$ and $\delta_{j} \approx 0$, then $v_{i}<\widehat{v}_{i}$. The source of this ambiguity is that all else being equal, while a more patient agent obtains a greater share when efforts have a persistent effect, he also incurs a greater effort cost. Which way this ambiguity is resolved thus depends on the other agent's relative impatience.

\section{Concluding remarks}

A fundamental task of economic analysis is to understand how resources are allocated between agents with conflicting preferences. This task becomes particularly challenging when complete contracts are unavailable. The sequential bargaining and rent-seeking literatures offer complementary frameworks to predict the allocation in the absence of complete contracts. In this paper, I have combined the insights from the two literatures to shed new light on many real negotiations where, as in the rent-seeking literature, players expend efforts to claim the rents associated with proposal power-a key prediction of the bargaining literature.

The analysis, however, has maintained some strong assumptions, and relaxing them might yield additional insights. For one, the recognition probabilities are assumed to take the specific functional form in A.2. Opening up this "black box" may provide further insights. For instance, in an interesting line of research, Perez-Castrillo and Wettstein [28,29] investigate the implementation of Shapley value through noncooperative behavior. In their mechanism, agents initially bid to be the proposer, and then the winner proposes how to share the coalition's surplus. However, it is important in their setup that bids be paid to nonproposers and be independent of subsequent sharing of the surplus. Although, in our setup, bids are wasted resources, one can imagine these bids given to a mediator, who is an unproductive agent. Moreover, a $k$-majority rule restricts the size of the coalition to achieve full production, and any smaller size coalition obtains zero surplus. In this more abstract framework, it would be interesting to see whether an agent's equilibrium 
payoff reflects a modified version of Shapley value. Second, it would be desirable to generalize the result on the socially optimal voting rule to an environment with heterogenous agents.

Aside from the extension in Section 6, our model can be fruitfully extended in various other ways. For instance, the present setting assumes that agents' efforts are unproductive and thus socially undesirable. It would be interesting to investigate a setting where agents allocate their resources between productive activities to increase the surplus and unproductive activities to propose. ${ }^{35}$ In addition, in many real world negotiations, agents form binding coalitions or "voting blocks". Using the insights from the recent literature on endogenous coalition formation, ${ }^{36}$ it would be useful to see the impact of costly recognition and voting rules on the equilibrium number and sizes of coalitions.

\section{Appendix A.}

I first note two useful results that hold in a one-shot rent-seeking game with exogenous prizes.

Lemma A1. In a one-shot rent-seeking game in which $p_{i}(\mathbf{x})$ satisfies A.1, $c_{i}=c$ for all $i \in N$, and the winner receives an exogenous prize of size 1, there exists a unique pure strategy equilibrium such that $x_{i}=x_{j}>0$ and $p_{i}(\mathbf{x})=p_{j}(\mathbf{x})=\frac{1}{n}$ for all $i, j \in N$.

Proof. Let $R_{i}\left(\mathbf{x}_{-i}\right)=\arg \max _{x_{i} \in\left[0, \frac{1}{c}\right]}\left[p_{i}\left(x_{i}, \mathbf{x}_{-i}\right)-c x_{i}\right]$ be agent $i$ 's reaction function. Given A.1, $R_{i}\left(\mathbf{x}_{-i}\right):\left[0, \frac{1}{c}\right]^{n-1} \rightarrow\left[0, \frac{1}{c}\right]$ is well-defined, and in particular continuous. Thus, standard fixed point arguments imply that there exists a pure strategy equilibrium. Observe that $\mathbf{x}=\mathbf{0}$ cannot be an equilibrium. Otherwise, given $\mathbf{x}_{-i}=\mathbf{0}$, agent $i$ could choose a small $x_{i}>0$ and receive the prize with probability 1 by part (b) of A.1. Next, note that in any equilibrium, $x_{i}>0$ for all $i$. Suppose, on the contrary, $x_{k}=0$ for some $k$. Also, let $x_{l}>0$ for some $l$ in the same equilibrium. From the FOCs it follows that $\frac{\partial p_{k}\left(0, x_{l}, \mathbf{x}_{-k, l}\right)}{\partial x_{k}} \leqslant c=\frac{\partial p_{l}\left(x_{l}, 0, \mathbf{x}_{-k, l}\right)}{\partial x_{l}}$. The symmetry assumption in A.1 implies that $\frac{\partial p_{k}\left(0, x_{l}, \mathbf{x}_{-k, l}\right)}{\partial x_{k}}=\frac{\partial p_{l}\left(0, x_{l}, \mathbf{x}_{-k, l}\right)}{\partial x_{l}}$, and hence $\frac{\partial p_{l}\left(0, x_{l}, \mathbf{x}_{-k, l}\right)}{\partial x_{l}} \leqslant \frac{\partial p_{l}\left(x_{l}, 0, \mathbf{x}_{-k, l}\right)}{\partial x_{l}}$. But this contradicts part (b) of A.1.

Now, take any two agents $i$ and $j$ and restrict attention to $\mathbf{x}>\mathbf{0}$. From the FOCs, reaction functions for $i$ and $j$ satisfy

$$
\frac{\partial p_{i}}{\partial x_{i}}\left(R_{i}\left(\mathbf{x}_{-i}\right), x_{j}, \mathbf{x}_{-i, j}\right)=c=\frac{\partial p_{j}}{\partial x_{j}}\left(R_{j}\left(\mathbf{x}_{-j}\right), x_{i}, \mathbf{x}_{-i, j}\right)
$$

This means reaction functions for $i$ and $j$ must be symmetric about the 45-degree line for any $\mathbf{x}_{-i, j}$, which implies that in equilibrium, $x_{i}=x>0$ and $p_{i}(\mathbf{x})=\frac{1}{n}$ for all $i$. To show uniqueness, note that $x$ solves $\frac{\partial p_{i}}{\partial x_{i}}(x, \ldots, x)=c$. Using parts (c) and (d) in A. 1 and $\sum_{i} p_{i}(\mathbf{x})=1$, it is easy to see that $\frac{\partial^{2} p_{i}}{\partial x_{i} \partial x_{j}}(x, \ldots, x)<0$ for $j \neq i$. Since $\frac{\partial^{2} p_{i}}{\partial x_{i}^{2}}(x, \ldots, x)<0$, it follows that $\frac{\partial p_{i}}{\partial x_{i}}(x, \ldots, x)$ is decreasing in $x$. Hence, the equilibrium must be unique.

\footnotetext{
${ }^{35}$ Skaperdas [34] studies a model in this direction but not in a bargaining framework.

${ }^{36}$ See, for instance, Bloch [6], Bloch et al. [7], Ray and Vohra [31,32], Maskin [21], and Okada [27].
} 
Lemma A2. In a one-shot rent-seeking game in which $p_{i}(\mathbf{x})$ satisfies A.2, and the winner receives an exogenous prize $\pi_{i}>0$, there exists a unique pure strategy equilibrium. Moreover, if $\frac{c_{i}}{\pi_{i}}<\frac{c_{j}}{\pi_{j}}$ for some $i, j \in N$, then $x_{i} \geqslant x_{j}$ and thus $p_{i}(\mathbf{x}) \geqslant p_{j}(\mathbf{x})$ (with strict inequality whenever $x_{i}>0$ ).

Proof. Szidarovski and Okuguchi [37] show the existence of a unique pure strategy equilibrium in a one-shot rent-seeking game with the following properties: (1) the winner receives a prize normalized to 1, (2) $p_{i}(\mathbf{x})$ satisfies A.2 with $f\left(x_{i}\right)=x_{i}$, and (3) the cost of effort is $g_{i}\left(x_{i}\right)$, where $g_{i}(0)=0, g_{i}^{\prime}>0$, and $g_{i}^{\prime \prime} \geqslant 0$. Moreover, $\mathbf{x}=\mathbf{0}$ cannot be an equilibrium. Now note that the one-shot contest described in Lemma A2 is equivalent to the contest in Szidarovski and Okuguchi [37], where $g_{i}\left(x_{i}\right)=f^{-1}\left(\frac{c_{i}}{\pi_{i}} x_{i}\right)$. Thus, there exists a unique pure strategy equilibrium.

Suppose $\frac{c_{i}}{\pi_{i}}<\frac{c_{j}}{\pi_{j}}$ for some $i, j \in N$, but, on the contrary, $x_{i}<x_{j}$. This implies $x_{j}>0$ and $\frac{c_{i}}{\pi_{i}} x_{i}<\frac{c_{j}}{\pi_{j}} x_{j}$. Moreover, since $f^{-1}($.$) is increasing and weakly convex, we have \frac{c_{i}}{\pi_{i}} f^{-1}\left(\frac{c_{i}}{\pi_{i}} x_{i}\right) \leqslant$ $\cdot \frac{c_{j}}{\pi_{j}} f^{-1 \prime}\left(\frac{c_{j}}{\pi_{j}} x_{j}\right)$. From the FOCs for efforts,

$$
\frac{\sum_{l \neq i} x_{l}}{\left(\sum_{l} x_{l}\right)^{2}}-\frac{c_{i}}{\pi_{i}} f^{-1 \prime}\left(\frac{c_{i}}{\pi_{i}} x_{i}\right) \leqslant 0=\frac{\sum_{l \neq j} x_{l}}{\left(\sum_{l} x_{l}\right)^{2}}-\frac{c_{j}}{\pi_{j}} f^{-1 \prime}\left(\frac{c_{j}}{\pi_{j}} x_{j}\right)
$$

or, equivalently

$$
0 \leqslant \frac{c_{j}}{\pi_{j}} f^{-1 \prime}\left(\frac{c_{j}}{\pi_{j}} x_{j}\right)-\frac{c_{i}}{\pi_{i}} f^{-1 \prime}\left(\frac{c_{i}}{\pi_{i}} x_{i}\right) \leqslant \frac{x_{i}-x_{j}}{\left(\sum_{l} x_{l}\right)^{2}}<0,
$$

a contradiction. Hence, $x_{i} \geqslant x_{j}$ and $p_{i}(\mathbf{x}) \geqslant p_{j}(\mathbf{x})$. Finally, if $x_{i}>0$, these inequalities must be strict. Otherwise, we would have $x_{i}=x_{j}>0$ for which, given $\frac{c_{i}}{\pi_{i}}<\frac{c_{j}}{\pi_{j}}$, the FOCs would yield a contradiction.

Proof of Proposition 1. Suppose $p_{i}(\mathbf{x})$ satisfies A.1, and $c_{i}=c$ for all $i \in N$. Let $\mathbf{v}$ be a stationary payoff vector. Since each agent has veto power, we can restrict attention to $\mathbf{v} \geqslant \mathbf{0}$. Moreover, by definition, we must have $\sum_{i} v_{i} \leqslant 1$ and thus $\pi^{n} \equiv 1-\sum_{i} \delta_{i} v_{i}>0$. Lemma A1 implies that there is a unique and symmetric solution to (3), i.e., $x_{i}=x\left(\pi^{n}, c\right)>0$, and $p_{i}=\frac{1}{n}$ for all $i \in N$ in equilibrium. Inserting this fact into (2) yields

$$
\left(1-\delta_{i}\right) v_{i}=\frac{1}{n} \pi^{n}-c x\left(\pi^{n}, c\right) .
$$

This immediately shows the last part. To prove the existence and uniqueness of $\mathbf{v}$, we rewrite (A.1): $\delta_{i} v_{i}=\frac{\delta_{i}}{1-\delta_{i}}\left[\frac{1}{n} \pi^{n}-c x\left(\pi^{n}, c\right)\right]$. Summing over both sides, we obtain $1-\pi^{n}=$ $\left[\frac{1}{n} \pi^{n}-c x\left(\pi^{n}, c\right)\right] \sum_{i} \frac{\delta_{i}}{1-\delta_{i}}$. Now define $F\left(\pi^{n}\right)=1-\pi^{n}-\left[\frac{1}{n} \pi^{n}-c x\left(\pi^{n}, c\right)\right] \sum_{i} \frac{\delta_{i}}{1-\delta_{i}}$ for $\pi^{n} \in$ $(0,1]$. Note $F(1) \leqslant 0$ and $\lim _{\pi^{n} \rightarrow 0} F\left(\pi^{n}\right)=1>0$. Moreover, it is well-established in the contest literature that the equilibrium expected net payoff in a one-shot game, $\frac{1}{n} \pi^{n}-c x\left(\pi^{n}, c\right)$, is weakly increasing in $\pi^{n}$, revealing that $F^{\prime}\left(\pi^{n}\right)<0$. Hence, there exists a unique solution to $F\left(\pi^{n}\right)=0$. Using this solution, Eq. (A.1) uniquely generates $\mathbf{v}$ as well as $\mathbf{x}$. 
Proof of Proposition 2. Suppose $p_{i}(\mathbf{x})$ satisfies A.2. Let $\mathbf{v} \geqslant 0$ be a stationary payoff vector. As in the previous proof, note $\pi^{n} \equiv 1-\sum_{i} \delta_{i} v_{i}>0$. Lemma A2 implies that there is a unique solution to (3), i.e., $x_{i}=x_{i}\left(\pi^{n}, c_{i}\right)$, with at least one agent with a strictly positive effort. Suppose $c_{i}<c_{j}$ for some $i, j \in N$. From Lemma A2, it follows that $x_{i} \geqslant x_{j}$ and thus $p_{i} \geqslant p_{j}$ in equilibrium. To show the last part, we substitute for $c_{i} x_{i}$ from (3) in (2) to obtain

$$
\left(1-\delta_{i}\right) v_{i}=\bar{p}_{i} \pi^{n} .
$$

Note that $\bar{p}_{i}(\mathbf{x}) \equiv\left(1-\varepsilon\left(x_{i}\right)\right) p_{i}(\mathbf{x})+\varepsilon\left(x_{i}\right) p_{i}^{2}(\mathbf{x})$ is decreasing in $\varepsilon\left(x_{i}\right)\left(\right.$ since $-p_{i}(\mathbf{x})+p_{i}^{2}(\mathbf{x})<$ 0 ) and increasing in $p_{i}(\mathbf{x})$. Given that $\varepsilon^{\prime}\left(x_{i}\right) \leqslant 0$ by A.2, it follows that $\bar{p}_{i}(\mathbf{x})$ is increasing in $x_{i}$. From (A.2), this implies $\left(1-\delta_{i}\right) v_{i} \geqslant\left(1-\delta_{j}\right) v_{j}$. To prove the existence and uniqueness of $\mathbf{v}$, we sum over (A.2) to obtain $1-\pi^{n}=\sum_{i} \frac{\delta_{i}}{1-\delta_{i}} \bar{p}_{i} \pi^{n}$, and define $G\left(\pi^{n}\right)=1-\pi^{n}-\sum_{i} \frac{\delta_{i}}{1-\delta_{i}} \bar{p}_{i} \pi^{n}$ for $\pi^{n} \in(0,1]$. Note $G(1) \leqslant 0$ and $G(0)>0$. Moreover, $\bar{p}_{i} \pi^{n}$ is the net payoff in a one-shot contest with a prize $\pi^{n}$, and it is well-known that this payoff is weakly increasing in the prize. Thus, we also have $G^{\prime}\left(\pi^{n}\right)<0$, implying a unique solution to $G\left(\pi^{n}\right)=0$. Using this solution, (A.2) uniquely identifies $\mathbf{v}$ and $\mathbf{x}$.

Proof of Lemma 1. Using (A.2) and recalling $\pi^{n}=1-\sum_{i} \delta_{i} v_{i}$, we observe that $\sum_{i} v_{i}=$ $\sum_{i} \frac{\bar{p}_{i}}{1-\delta_{i}} \pi^{n}$ and $\pi^{n}=\frac{1}{1+\sum_{i} \frac{\delta_{i}}{1-\delta_{i}} \bar{p}_{i}}$. Since $S C=\sum_{i} c_{i} x_{i}=1-\sum_{i} v_{i}$, we obtain the expression in (7).

Proof of Proposition 3. Suppose $p_{i}(\mathbf{x})$ satisfies A.2. To prove the first part, let $c_{i}=c$ for all $i$. From Proposition 1, this implies $p_{i}=\frac{1}{n}$. Inserting this fact into (7) yields

$$
S C=n c x=\frac{n(n-1) \varepsilon(x)}{n(n-1) \varepsilon(x)+[n(1-\varepsilon(x))+\varepsilon(x)] \sum_{i} \frac{1}{1-\delta_{i}}} .
$$

Let $\delta^{\prime}=\left(\delta_{1}^{\prime}, \ldots, \delta_{n}^{\prime}\right)$ be a mean-preserving spread of $\delta^{\prime \prime}=\left(\delta_{1}^{\prime \prime}, \ldots, \delta_{n}^{\prime \prime}\right)$ such that (1) $\sum_{i} \delta_{i}^{\prime}=$ $\sum_{i} \delta_{i}^{\prime \prime}$ and (2) $\delta_{j}^{\prime} \geqslant \delta_{j}^{\prime \prime}$ if and only if $\delta_{j}^{\prime \prime} \geqslant \frac{1}{n} \sum_{i} \delta_{i}^{\prime \prime}$ for $j \in N$. I argue that $\sum_{i} \frac{1}{1-\delta_{i}^{\prime}}>\sum_{i} \frac{1}{1-\delta_{i}^{\prime \prime}}$. To do so, I utilize second-order stochastic dominance arguments by defining a random variable, $\widetilde{\delta}$, such that $P\left\{\widetilde{\delta}=\delta_{i}\right\}=\frac{1}{n}$. Note that $\widetilde{\delta}^{\prime \prime}$ second-order stochastically dominates $\widetilde{\delta}^{\prime}$ if and only if $\delta^{\prime}$ is a mean-preserving spread of $\delta^{\prime \prime}$. Moreover, since $h\left(\delta_{i}\right)=\frac{1}{1-\delta_{i}}$ is a convex function, it follows that $E_{\widetilde{\delta}^{\prime}}\left[h\left(\widetilde{\delta}^{\prime}\right)\right]>E_{\widetilde{\delta}^{\prime \prime}}\left[h\left(\widetilde{\delta}^{\prime \prime}\right)\right]$, or equivalently $\sum_{i} \frac{1}{1-\delta_{i}^{\prime}}>\sum_{i} \frac{1}{1-\delta_{i}^{\prime \prime}}$. Now, suppose, on the contrary, that $x^{\prime} \geqslant x^{\prime \prime}$. This implies $S C^{\prime} \geqslant S C^{\prime \prime}$ and $\varepsilon\left(x^{\prime}\right) \leqslant \varepsilon\left(x^{\prime \prime}\right)$. Since $S C$ is increasing in $\varepsilon(x)$ and decreasing in $\sum_{i} \frac{1}{1-\delta_{i}}$, it must be that $S C^{\prime}<S C^{\prime \prime}$, a contradiction. Hence $x^{\prime}<x^{\prime \prime}$ and $S C^{\prime}<S C^{\prime \prime}$.

To prove the second part, suppose $\delta_{i}=\delta$ for all $i$ and $f\left(x_{i}\right)=x_{i}$. This implies $\varepsilon\left(x_{i}\right)=1$ and hence $\bar{p}_{i}=p_{i}^{2}$. Using (7), we find that $p_{i}=1-(n-1) \frac{c_{i}}{\sum_{j \in P^{+}} c_{j}}$ where $P^{+}=\left\{i \in N \mid p_{i}>0\right\}$.

Now, let $c^{\prime}=\left(c_{1}^{\prime}, \ldots, c_{n}^{\prime}\right)$ be a mean-preserving spread of $c^{\prime \prime}=\left(c_{1}^{\prime \prime}, \ldots, c_{n}^{\prime \prime}\right)$, which means $p^{\prime}$ is a mean-preserving spread of $p^{\prime \prime}$. Define the random variable $\widetilde{p}$ such that $\operatorname{Pr}\left\{\tilde{p}=p_{i}\right\}=\frac{1}{n}$. Since 
$g\left(p_{i}\right)=p_{i}^{2}$ is a strictly convex function, $E_{\widetilde{p}^{\prime \prime}}\left[g\left(\widetilde{p}^{\prime \prime}\right)\right]<E_{\widetilde{p}^{\prime}}\left[g\left(\widetilde{p}^{\prime}\right)\right]$ or, equivalently $\sum_{i} p_{i}{ }^{\prime \prime 2}<$ $\sum_{i} p_{i}^{\prime 2}$. Finally, given that $S C$ is decreasing in $\sum_{i} p_{i}^{2}$, it follows that $S C^{\prime}<S C^{\prime \prime}$.

To prove the last part, let $c_{i}=c$ and $\delta_{i}=\delta$ for all $i$. Differentiating both sides of (A.3), we first note that $x$ is decreasing, and hence $\varepsilon(x)$ is increasing in $n$. Given that $S C$ is increasing in $n$ and $\varepsilon(x)$, the result follows.

Proof of Lemma 2. Suppose the voting rule is $k$-majority and $p_{i}(\mathbf{x})$ satisfies A.2. Moreover, suppose, in equilibrium, $\delta_{j} v_{j}<\delta_{i} v_{i}$ for some $i, j \in N$. Recall that $\pi_{i}^{k} \equiv 1-w_{i}-\frac{\mu_{i}}{1-p_{i}} \delta_{i} v_{i}$, where $\mu_{i} \equiv \sum_{j \neq i} p_{j} \psi_{j i}$. Let $\delta_{k} v_{k}$ be the $k$ th smallest continuation value in equilibrium (or, equivalently, the $k$ th cheapest vote) and also let $w_{k}$ be the payment such a player makes. In general, the following has to hold in equilibrium:

$$
\mu_{i} \begin{cases}=1-p_{i} & \text { if } \delta_{i} v_{i}<\delta_{k} v_{k}, \\ \leqslant 1-p_{i} & \text { if } \delta_{i} v_{i}=\delta_{k} v_{k} \\ =0 & \text { if } \delta_{i} v_{i}>\delta_{k} v_{k}\end{cases}
$$

and

$$
w_{i}= \begin{cases}w_{k}+\delta_{k} v_{k}-\delta_{i} v_{i} & \text { if } \delta_{i} v_{i} \leqslant \delta_{k} v_{k} \\ w_{k} & \text { if } \delta_{i} v_{i} \geqslant \delta_{k} v_{k}\end{cases}
$$

Now consider the following three cases. First, suppose $\delta_{j} v_{j}<\delta_{i} v_{i}<\delta_{k} v_{k}$. From (A.4), this implies $\mu_{i}=1-p_{i}$ and $\mu_{j}=1-p_{j}$. Furthermore, since $\delta_{i} v_{i}<\delta_{k} v_{k}$ and $\delta_{j} v_{j}<\delta_{k} v_{k}$, from (A.5), we have $w_{i}=w_{k}+\delta_{k} v_{k}-\delta_{i} v_{i}$ and $w_{j}=w_{k}+\delta_{k} v_{k}-\delta_{j} v_{j}$. Together these facts reveal that $\pi_{i}^{k}=\pi_{j}^{k}=1-w_{k}-\delta_{k} v_{k}$. Second, suppose $\delta_{k} v_{k}<\delta_{j} v_{j}<\delta_{i} v_{i}$. Once again, using (A.4) and (A.5), this implies $\mu_{i}=\mu_{j}=0$ and $w_{i}=w_{j}=w_{k}$, which in turn imply $\pi_{i}^{k}=\pi_{j}^{k}=\pi-w_{k}$. Finally, suppose $\delta_{j} v_{j} \leqslant \delta_{k} v_{k} \leqslant \delta_{i} v_{i}$ (with at least one inequality being strict). From (A.5), we have $w_{i}=w_{k}$ and $w_{j}=w_{k}+\delta_{k} v_{k}-\delta_{j} v_{j}$. Furthermore, given $\mu_{i}=0$ whenever $\delta_{k} v_{k}<\delta_{i} v_{i}$, we have $\pi_{i}^{k}=1-w_{k}-\frac{\mu_{i}}{1-p_{i}} \delta_{i} v_{i} \geqslant 1-w_{k}-\frac{\mu_{i}}{1-p_{i}} \delta_{k} v_{k}$, and

$$
\begin{aligned}
\pi_{j}^{k} & =1-w_{k}-\delta_{k} v_{k}+\delta_{j} v_{j}-\frac{\mu_{j}}{1-p_{j}} \delta_{j} v_{j} \\
& =1-w_{k}-\delta_{k} v_{k}+\left(1-\frac{\mu_{j}}{1-p_{j}}\right) \delta_{j} v_{j} \\
& \leqslant 1-w_{k}-\delta_{k} v_{k}+\left(1-\frac{\mu_{j}}{1-p_{j}}\right) \delta_{k} v_{k} \\
& =1-w_{k}-\frac{\mu_{j}}{1-p_{j}} \delta_{k} v_{k} .
\end{aligned}
$$

Since $\delta_{j} v_{j}<\delta_{i} v_{i}$ by hypothesis, we also have $\frac{\mu_{i}}{1-p_{i}} \leqslant \frac{\mu_{j}}{1-p_{j}}$ and hence $\pi_{i}^{k} \geqslant \pi_{j}^{k}$. Overall, we have shown that if, in equilibrium, $\delta_{j} v_{j}<\delta_{i} v_{i}$ for some $i, j \in N$, then $\pi_{j}^{k} \leqslant \pi_{i}^{k}$.

To prove the second part, suppose $\delta_{j} \leqslant \delta_{i}$ and $c_{i} \leqslant c_{j}$, and, in equilibrium, $\delta_{j} v_{j}=\delta_{i} v_{i}$ for some $i, j \in N$. By way of contradiction, assume $\pi_{i}^{k}<\pi_{j}^{k}$. Using a similar argument to the first part, it easily follows that $\pi_{j}^{k}=\pi_{i}^{k}$ whenever $\delta_{j} v_{j}=\delta_{i} v_{i}<\delta_{k} v_{k}$ or $\delta_{k} v_{k}<\delta_{j} v_{j}=\delta_{i} v_{i}$, yielding a contradiction. Now, consider the case in which $\delta_{j} v_{j}=\delta_{k} v_{k}=\delta_{i} v_{i}$. This means $w_{j}=w_{i}=w_{k}$, and given $\delta_{j} \leqslant \delta_{i}, v_{i} \leqslant v_{j}$. Now, using the expression in (A.7) below, we also 
have $v_{i}=1-w_{k}-\left(1-\bar{p}_{i}\right) \pi_{i}^{k}$ and $v_{j}=1-w_{k}-\left(1-\bar{p}_{j}\right) \pi_{j}^{k}$. Since $v_{i} \leqslant v_{j}$, we must have $\left(1-\bar{p}_{i}\right) \pi_{i}^{k} \geqslant\left(1-\bar{p}_{j}\right) \pi_{j}^{k}$. Furthermore, since $\pi_{i}^{k}<\pi_{j}^{k}$ by hypothesis, we must also have $\bar{p}_{i}<\bar{p}_{j}$, and hence $x_{i}<x_{j}$. This means $x_{j}>0$. Using the FOCs in (11), we obtain

$$
\varepsilon_{i} \frac{f\left(x_{i}\right) / x_{i}}{\sum_{l} f\left(x_{l}\right)}\left(1-p_{i}\right) \pi_{i}^{k} \leqslant c_{i} \leqslant c_{j}=\varepsilon_{j} \frac{f\left(x_{j}\right) / x_{j}}{\sum_{l} f\left(x_{l}\right)}\left(1-p_{j}\right) \pi_{j}^{k} .
$$

Since $f\left(x_{i}\right) / x_{i} \leqslant f\left(x_{j}\right) / x_{j}$ and $\varepsilon_{i} \geqslant \varepsilon_{j}$, we have $\left(1-p_{i}\right) \pi_{i}^{k} \leqslant\left(1-p_{j}\right) \pi_{j}^{k}$. Moreover, since $c_{i} \leqslant c_{j}$ and $\varepsilon_{i} p_{i}\left(1-p_{i}\right)=-\left(1-p_{i}\right)+1-\bar{p}_{i}$ by definition, we also have

$$
\left[-\left(1-p_{i}\right)+1-\bar{p}_{i}\right] \pi_{i}^{k} \leqslant c_{i} x_{i}<c_{j} x_{j}=\left[-\left(1-p_{j}\right)+1-\bar{p}_{j}\right] \pi_{j}^{k}
$$

and hence $\left(1-\bar{p}_{i}\right) \pi_{i}^{k}<\left(1-\bar{p}_{j}\right) \pi_{j}^{k}$, contradicting $\left(1-\bar{p}_{i}\right) \pi_{i}^{k} \geqslant\left(1-\bar{p}_{j}\right) \pi_{j}^{k}$, revealing that $\pi_{i}^{k} \geqslant \pi_{j}^{k}$.

Proof of Proposition 4. First, we characterize the equilibrium, and then show that there exists one with a unique $(\mathbf{x}, \mathbf{v})$ pair. Inserting (11) into (10),

$$
\begin{aligned}
& v_{i}=\bar{p}_{i}\left(1-w_{i}\right)+\left(1+\varepsilon_{i} p_{i}\right) \mu_{i} \delta_{i} v_{i}, \quad \text { or equivalently } \\
& v_{i}=1-w_{i}-\left(1-\bar{p}_{i}\right) \pi_{i}^{k} .
\end{aligned}
$$

Let $\delta_{j} \leqslant \delta_{i}$ and $c_{i} \leqslant c_{j}$ for some $i, j \in N$ and suppose, by way of contradiction, that $\delta_{i} v_{i}<\delta_{j} v_{j}$ in equilibrium. We consider three relevant cases and generate a contradiction in each case.

Case 1: $\delta_{k} v_{k}<\delta_{i} v_{i}<\delta_{j} v_{j}$. Then, from (A.4) and (A.5), $w_{i}=w_{j}=w_{k}$ and $\mu_{i}=\mu_{j}=0$, which yield $\pi_{i}^{k}=\pi_{j}^{k}=1-w_{k}$. This implies $x_{i} \geqslant x_{j}$ by Lemma A2, which, in turn, implies $\bar{p}_{i} \geqslant \bar{p}_{j}$ and $v_{i} \geqslant v_{j}$ by (A.6). Given $\delta_{j} \leqslant \delta_{i}$, we have $\delta_{j} v_{j} \leqslant \delta_{i} v_{i}$, yielding a contradiction.

Case 2: $\delta_{i} v_{i}<\delta_{j} v_{j}<\delta_{k} v_{k}$. From (A.4), we have $\mu_{i}=1-p_{i}$ and $\mu_{j}=1-p_{j}$, revealing that $\pi_{i}^{k}=\pi_{j}^{k}=1-w_{k}-\delta_{k} v_{k}$ and hence $x_{i} \geqslant x_{j}$ by Lemma A2. This implies $\bar{p}_{i} \geqslant \bar{p}_{j}$. From here, given $\delta_{j} \leqslant \delta_{i}$ and (A.7)

$$
v_{j}=\frac{\bar{p}_{j} \pi_{j}^{k}}{1-\delta_{j}} \leqslant \frac{\bar{p}_{i} \pi_{i}^{k}}{1-\delta_{i}}=v_{i}
$$

implying $\delta_{j} v_{j} \leqslant \delta_{i} v_{i}-$ a contradiction.

Case 3: $\delta_{i} v_{i} \leqslant \delta_{k} v_{k} \leqslant \delta_{j} v_{j}$ (at least one inequality being strict). Since $\delta_{i} v_{i}<\delta_{j} v_{j}$ and $\delta_{j} \leqslant \delta_{i}$, we have $v_{i}<v_{j}$. Moreover, Lemma 2 implies $\pi_{i}^{k} \leqslant \pi_{j}^{k}$. Now, I argue that $x_{i} \leqslant x_{j}$. Suppose not. Then, $x_{i}>x_{j}$ and hence $\bar{p}_{i}>\bar{p}_{j}$. Given $\pi_{i}^{k} \leqslant \pi_{j}^{k}$, this implies $\left(1-\bar{p}_{i}\right) \pi_{i}^{k}<\left(1-\bar{p}_{j}\right) \pi_{j}^{k}$. From (A.7), we must then have

$$
v_{i}+w_{i}=1-\left(1-\bar{p}_{i}\right) \pi_{i}^{k}>1-\left(1-\bar{p}_{j}\right) \pi_{j}^{k}=v_{j}+w_{j} .
$$

Note that (A.5) reveals that $w_{i}=w_{k}+\delta_{k} v_{k}-\delta_{i} v_{i}$ and $w_{j}=w_{k}$. Inserting these facts into (A.8) and canceling terms reveal that $v_{j}<\delta_{k} v_{k}+\left(1-\delta_{i}\right) v_{i}$. Since $\delta_{k} v_{k} \leqslant \delta_{j} v_{j}$, this further reveals $v_{j}<\delta_{j} v_{j}+\left(1-\delta_{i}\right) v_{i}$, or equivalently $\left(1-\delta_{j}\right) v_{j}<\left(1-\delta_{i}\right) v_{i}$. Given $\delta_{j} \leqslant \delta_{i}$ and $v_{i}<v_{j}$ by hypothesis, this yields a contradiction. Hence, $x_{i} \leqslant x_{j}$.

This means $p_{i} \leqslant p_{j}$ and $\varepsilon_{i} \geqslant \varepsilon_{j}$. Note that $x_{j}>0$. Otherwise, we would have $x_{j}=x_{i}=0$, which would imply $v_{i}=v_{j}=0$, and contradict $\delta_{i} v_{i}<\delta_{j} v_{j}$. Moreover, using the FOCs in (11), and the exact arguments as in the last part of the proof of Lemma 2, it follows that $\left(1-\bar{p}_{i}\right) \pi_{i}^{k} \leqslant\left(1-\bar{p}_{j}\right) \pi_{j}^{k}$, where weak inequality follows because $x_{i} \leqslant x_{j}$. 
Once again, using (A.5), we observe $w_{i}=w_{k}+\delta_{k} v_{k}-\delta_{i} v_{i}$ and $w_{j}=w_{k}$. Furthermore, from (A.7), we have $v_{i}=\frac{1-w_{k}-\delta_{k} v_{k}-\left(1-\bar{p}_{i}\right) \pi_{i}^{k}}{1-\delta_{i}}$ and $v_{j}=1-w_{k}-\left(1-\bar{p}_{j}\right) \pi_{j}^{k}$, respectively. Using these facts and $\delta_{i} v_{i} \leqslant \delta_{k} v_{k}$ by hypothesis reveals

$$
\begin{aligned}
& \frac{\delta_{i}\left[1-w_{k}-\delta_{k} v_{k}-\left(1-\bar{p}_{i}\right) \pi_{i}^{k}\right]}{1-\delta_{i}} \leqslant \delta_{k} v_{k} \\
& \quad \Longrightarrow \delta_{i}\left[1-w_{k}-\left(1-\bar{p}_{i}\right) \pi_{i}^{k}\right] \leqslant \delta_{k} v_{k}=\delta_{k}\left[1-w_{k}-\left(1-\bar{p}_{k}\right) \pi_{k}^{k}\right] \\
& \quad \Longrightarrow\left(\delta_{i}-\delta_{k}\right)\left(1-w_{k}\right) \leqslant \delta_{i}\left(1-\bar{p}_{i}\right) \pi_{i}^{k}-\delta_{k}\left(1-\bar{p}_{k}\right) \pi_{k}^{k} .
\end{aligned}
$$

Moreover, given $\delta_{k} v_{k} \leqslant \delta_{j} v_{j}$,

$$
\begin{aligned}
\delta_{k}\left[1-w_{k}-\left(1-\bar{p}_{k}\right) \pi_{k}^{k}\right] & \leqslant \delta_{j}\left[1-w_{k}-\left(1-\bar{p}_{j}\right) \pi_{j}^{k}\right] \\
& \Longrightarrow\left(\delta_{k}-\delta_{j}\right)\left(1-w_{k}\right) \leqslant \delta_{k}\left(1-\bar{p}_{k}\right) \pi_{k}^{k}-\delta_{j}\left(1-\bar{p}_{j}\right) \pi_{j}^{k} .
\end{aligned}
$$

Summing (A.9) and (A.10), we obtain

$$
\left(\delta_{i}-\delta_{j}\right)\left(1-w_{k}\right)<\delta_{i}\left(1-\bar{p}_{i}\right) \pi_{i}^{k}-\delta_{j}\left(1-\bar{p}_{j}\right) \pi_{j}^{k},
$$

where the strict inequality follows from the hypothesis of Case 3 . Since $\left(1-\bar{p}_{i}\right) \pi_{i}^{k} \leqslant\left(1-\bar{p}_{j}\right) \pi_{j}^{k}$, this further yields

$$
\begin{aligned}
\left(\delta_{i}-\delta_{j}\right)\left(1-w_{k}\right) & <\left(\delta_{i}-\delta_{j}\right)\left(1-\bar{p}_{i}\right) \pi_{i}^{k} \\
& \Longrightarrow 1-w_{k}<\left(1-\bar{p}_{i}\right) \pi_{i}^{k} \leqslant \pi_{i}^{k} \leqslant 1-w_{k},
\end{aligned}
$$

a contradiction.

Overall, the three cases reveal that $\delta_{j} v_{j} \leqslant \delta_{i} v_{i}$, proving the first part. Lemma 2 further implies $\pi_{j}^{k} \leqslant \pi_{i}^{k}$. Using Lemma A2, the desired result in the second part follows.

Using this characterization, I now show there exists an SSP equilibrium with a unique $(\mathbf{x}, \mathbf{v})$ pair. Since we have already shown the result for $k=n$ and the result trivially follows for $k=1$, I restrict attention to cases where $n \geqslant 3$ and $1<k<n$. Without loss of generality, let $\delta_{1} \leqslant \cdots \leqslant \delta_{k} \leqslant \cdots \leqslant \delta_{n}$ and $c_{1} \leqslant \cdots \leqslant c_{k} \leqslant \cdots \leqslant c_{n}$. In equilibrium, this implies $\delta_{1} v_{1} \leqslant \cdots \leqslant$ $\delta_{k} v_{k} \leqslant \cdots \leqslant \delta_{n} v_{n}$. Given this ordering, it is clear that each player $i$ must belong to one of the following four disjoint sets: For some $j_{0} \in\{1, \ldots, k\}$ and $j_{1} \in\{k, \ldots, n\}$,

$$
\begin{aligned}
& \Omega_{L}=\left\{i \in N \mid 1 \leqslant i \leqslant j_{0}-1 \text { and } \delta_{i} v_{i}<\delta_{k} v_{k}\right\}, \\
& \Omega_{E}=\left\{i \in N \mid j_{0} \leqslant i \leqslant k \text { and } \delta_{i} v_{i}=\delta_{k} v_{k}\right\}, \\
& \Lambda_{E}=\left\{i \in N \mid k \leqslant i \leqslant j_{1} \text { and } \delta_{i} v_{i}=\delta_{k} v_{k}\right\}, \\
& \Lambda_{H}=\left\{i \in N \mid j_{1}+1 \leqslant i \leqslant n \text { and } \delta_{i} v_{i}>\delta_{k} v_{k}\right\} .
\end{aligned}
$$

Suppose that $\Omega_{E}=\Lambda_{E}=\phi$. From (A.4) and (A.5), this implies that

$$
\pi_{i}= \begin{cases}1-w_{k}-\delta_{k} v_{k} \equiv \pi_{L} & \text { if } i<k, \\ 1-w_{k}-\frac{\sum_{j=1}^{k-1} p_{i}}{\sum_{j \neq k}^{n} p_{i}} \delta_{k} v_{k} & \text { if } i=k, \\ 1-w_{k} \equiv \pi_{H} & \text { if } i>k .\end{cases}
$$

Let $\tilde{\pi}_{i} \equiv \frac{c_{i}}{\pi_{i}}$. Eq. (A.11) together with (11) and Lemma A2 reveal that there exists unique efforts such that $x_{i} \stackrel{\pi_{i}}{=} x\left(\widetilde{\pi}_{i}, \tilde{\pi}_{-i}\right)$. Since $p_{i}(\mathbf{x})$ is symmetric, so are $x\left(\tilde{\pi}_{i}, \tilde{\pi}_{-i}\right)$ and $p_{i}=\phi\left(\tilde{\pi}_{i}, \tilde{\pi}_{-i}\right)$. Note 
from (A.11) that, in equilibrium, $\pi_{k}^{*}\left(\pi_{H}, \pi_{L}\right)$ solves $G\left(\pi_{k} \mid \pi_{H}, \pi_{L}\right)=0$, where

$$
G_{1}\left(\pi_{k} \mid \pi_{H}, \pi_{L}\right) \equiv \pi_{H}-\pi_{k}-\frac{\sum_{j=1}^{k-1} \phi\left(\tilde{\pi}_{i}, \tilde{\pi}_{-i}\right)}{\sum_{j \neq k}^{n} \phi\left(\tilde{\pi}_{i}, \tilde{\pi}_{-i}\right)}\left(\pi_{H}-\pi_{L}\right) .
$$

Observe that $G_{1}\left(\pi_{L} \mid \pi_{H}, \pi_{L}\right)>0$ and $G_{1}\left(\pi_{H} \mid \pi_{H}, \pi_{L}\right)<0$. Thus, there is $\pi_{k}^{*}\left(\pi_{H}, \pi_{L}\right) \in$ $\left[\pi_{L}, \pi_{H}\right]$ that solves $G_{1}\left(\pi_{k} \mid \pi_{H}, \pi_{L}\right)=0$. Moreover, somewhat complicated algebra reveals that $G_{1}^{\prime}\left(\pi_{k}^{*}\left(\pi_{H}, \pi_{L}\right) \mid \pi_{H}, \pi_{L}\right)<0$, which implies the uniqueness of $\pi_{k}^{*}\left(\pi_{H}, \pi_{L}\right)$. (Otherwise, if there were another solution in $\left[\pi_{L}, \pi_{H}\right]$, then $G_{1}^{\prime}() \geqslant$.0 would hold.)

Next, using (A.7) and (A.5), and inserting in $\pi_{k}^{*}\left(\pi_{H}, \pi_{L}\right)$, we note that, equilibrium, $\pi_{L}^{*}\left(\pi_{H}\right)$ solves $G_{2}\left(\pi_{L} \mid \pi_{H}\right)=0$ where

$$
G_{2}\left(\pi_{L} \mid \pi_{H}\right) \equiv \frac{\pi_{H}-\pi_{L}}{\delta_{k}}-\left[\bar{p}_{k} \pi_{H}+\left(1-\bar{p}_{k}\right) \frac{\sum_{j=1}^{k-1} \phi\left(\tilde{\pi}_{i}, \tilde{\pi}_{-i}\right)}{\sum_{j \neq k}^{n} \phi\left(\tilde{\pi}_{i}, \tilde{\pi}_{-i}\right)}\left(\pi_{H}-\pi_{L}\right)\right] .
$$

Since $G_{2}\left(0 \mid \pi_{H}\right)>0$ and $G_{2}\left(\pi_{H} \mid \pi_{H}\right)<0$, there is $\pi_{L}^{*}\left(\pi_{H}\right) \in\left[0, \pi_{H}\right]$ that solves $G_{2}\left(\pi_{L} \mid \pi_{H}\right)=$ 0 . Furthermore, $G_{2}^{\prime}\left(\pi_{L}^{*}\left(\pi_{H}\right) \mid \pi_{H}\right)<0$, which means $\pi_{L}^{*}\left(\pi_{H}\right)$ is unique. Finally, inserting the fact that $w_{i}=w_{k}+\delta_{k} v_{k}-\delta_{i} v_{i}$ for $i<k$ into (A.7) implies

$$
\delta_{i} v_{i}=\frac{\bar{p}_{i}}{1-\delta_{i}} \pi_{L}^{*}\left(\pi_{H}\right)
$$

Summing over both sides of (A.12), we obtain $w_{k}=\sum_{i=1}^{k-1} \frac{\bar{p}_{i}}{1-\delta_{i}} \pi_{L}^{*}\left(\pi_{H}\right)$. Since $w_{k}=1-\pi_{H}$, the equilibrium $\pi_{H}^{*}$ solves $G_{3}\left(\pi_{H}\right)=0$, where

$$
G_{3}\left(\pi_{H}\right)=1-\pi_{H}-\sum_{i=1}^{k-1} \frac{\bar{p}_{i}}{1-\delta_{i}} \pi_{L}^{*}\left(\pi_{H}\right) .
$$

Note that $G_{3}(0)=1>0$ and $G_{3}(1)<0$. Thus, there is $\pi_{H}^{*} \in(0,1)$ that solves $G_{3}\left(\pi_{H}\right)=0$. Furthermore, $G_{3}^{\prime}\left(\pi_{H}^{*}\right)<0$, implying that $\pi_{H}^{*}$ is unique. Given that the pair $(\mathbf{x}, \mathbf{v})$ is uniquely identified by $\pi_{i}$ 's, it also exists and is unique. However, for this to be part of an equilibrium, the conditions in $\Omega_{L}$ and $\Lambda_{H}$ that we have assumed have to be satisfied, or simply it must be that $\delta_{k-1} v_{k-1}<\delta_{k} v_{k}<\delta_{k+1} v_{k+1}$. If at least one of these conditions does not hold for specific $\delta_{i}$ 's, then it must be that the set $\Omega_{H}$ and/or $\Lambda_{E}$ is nonempty. Suppose $\Omega_{H}=\phi$ and $\Lambda_{E} \neq \phi$. Once again, define $\pi_{L}$ and $\pi_{H}$ as in (A.11). Since for $i \in \Lambda_{E}, \delta_{i} v_{i}=\delta_{k} v_{k}$, we have $v_{i}=\frac{\pi_{H}-\pi_{L}}{\delta_{i}}$ by (A.11). Furthermore, Eq. (A.7) implies that for $i \in\left\{k, \ldots, j_{1}\right\}, v_{i}=1-w_{k}-\left(1-\bar{p}_{i}\right) \pi_{i}$, or equivalently $v_{i}=\pi_{H}-\left(1-\bar{p}_{i}\right) \pi_{i}$. Substituting for $v_{i}=\frac{\pi_{H}-\pi_{L}}{\delta_{i}}$ reveals

$$
\frac{\pi_{H}-\pi_{L}}{\delta_{i}}=\pi_{H}-\left(1-\bar{p}_{i}\right) \pi_{i} \quad \text { for } i \in\left\{k, \ldots, j_{1}\right\}
$$

Let $\left(\pi_{k}\left(\pi_{L}, \pi_{H}\right), \ldots, \pi_{j_{1}}\left(\pi_{L}, \pi_{H}\right)\right)$ be the solution to (A.14). Recall $v_{k}=\pi_{H}-\left(1-\bar{p}_{k}\left(\pi_{L}, \pi_{H}\right)\right)$ $\pi_{k}\left(\pi_{L}, \pi_{H}\right)$. Given $\delta_{k} v_{k}=\pi_{H}-\pi_{L}$ by definition, the equilibrium $\pi_{L}^{*}\left(\pi_{H}\right)$ solves $G_{4}\left(\pi_{L} \mid \pi_{H}\right)=0$, 
where $G_{4}\left(\pi_{L} \mid \pi_{H}\right) \equiv\left[\left(1-\delta_{k}\right) \pi_{H}+\delta_{k}\left(1-\bar{p}_{k}\left(\pi_{L}, \pi_{H}\right)\right) \pi_{k}\left(\pi_{L}, \pi_{H}\right)\right]-\pi_{L}$. Since $G_{4}\left(0 \mid \pi_{H}\right)>0$ and $G_{4}\left(\pi_{H} \mid \pi_{H}\right)<0$, there is $\pi_{L}^{*}\left(\pi_{H}\right)$ solves $G_{4}\left(\pi_{L} \mid \pi_{H}\right)=0$. Moreover, since $G_{4}^{\prime}\left(\pi_{L}^{*}\left(\pi_{H}\right) \mid \pi_{H}\right)$ $<0$, it is unique. Finally, inserting $\pi_{L}^{*}\left(\pi_{H}\right)$ and using the same manipulations, we see that the equilibrium $\pi_{H}^{*} \in[0,1]$ must satisfy $G_{5}\left(\pi_{H}\right)=0$, where $G_{5}\left(\pi_{H}\right) \equiv 1-\pi_{H}-\sum_{i=1}^{k-1} \frac{\bar{p}_{i}}{1-\delta_{i}} \pi_{L}^{*}\left(\pi_{H}\right)$.

Using similar arguments as above, it is easy but tedious to show that there is a unique $\pi_{H}^{*} \in[0,1]$, which in turn uniquely determines the pair $(\mathbf{x}, \mathbf{v})$. For this to be part of an equilibrium, it must be that $\delta_{k-1} v_{k-1}<\delta_{k} v_{k}=\cdots=\delta_{j_{1}} v_{j_{1}}<\delta_{j_{1}+1} v_{j_{1}+1}$. If this condition is not satisfied, then we similarly exhaust the remaining possibilities for which we find a unique pair of $(\mathbf{x}, \mathbf{v})$. The existence of an equilibrium follows by construction.

Proof of Proposition 5. Suppose that $c_{i}=c$ and $\delta_{i}=\delta$ for all $i \in N$, and that $p_{i}(\mathbf{x})$ satisfies A.2. From Proposition 4 , the equilibrium pair of $(\mathbf{x}, \mathbf{v})$ is unique and symmetric. Inserting the facts $x_{i}=x, p_{i}=\frac{1}{n}, \pi_{i}^{r}=1-\delta r n v$, and $v=\frac{1}{n}-c x$ into (11) and solving for $v$ reveal

$$
\begin{aligned}
& v=\frac{1-\varepsilon(x)+\frac{\varepsilon(x)}{n}}{n-\delta r \varepsilon(x)(n-1)}, \\
& x=\frac{\varepsilon(x)(n-1)(1-\delta r)}{c n[n-\delta r \varepsilon(x)(n-1)]} .
\end{aligned}
$$

Suppose that $x$ weakly increases in $r$. This implies $\varepsilon(x)$ weakly decreases in $r$, and given that the r.h.s. of (A.15) increases in $\varepsilon(x)$ and decreases in $r$, so does the r.h.s. of (A.15), a contradiction. Hence, $x$ decreases in $r$. Similar arguments also reveal that $x$ decreases in $\delta$. Since $v=\frac{1}{n}-c x$, these imply that $v$ increases in $r$ and $\delta$.

To show that the total equilibrium cost, i.e., $n c x$ increases in $n$, suppose not. Then, $x$ must be decreasing in $n$, which means $\varepsilon(x)$ is increasing in $n$. Since $n c x=\frac{\varepsilon(x)(n-1)(1-\delta r)}{n-\delta r \varepsilon(x)(n-1)}$, and the r.h.s. is increasing in $n$ and $\varepsilon(x)$, the r.h.s. must be decreasing in $n$. This contradicts the 1.h.s. Hence, $n c x$ increases in $n$. Recall that $v=\frac{1}{n}-c x=\frac{1-n c x}{n}$, which means $v$ decreases in $n$.

Proof of Corollary 1. Recalling that $\Delta \equiv s_{p}-s_{n p}=1-\delta r n v$, and that, from the proof of Proposition 5, $n v$ is decreasing in $n$, the desired result follows.

Proof of Proposition 6. Suppose that $\delta_{i}=\delta$ and $c_{i}=c$ for all $i$ and that $p_{i}(\mathbf{x})$ and $q_{i}(\mathbf{x})$ satisfy A.2 and A. 3 with $\lambda \in(0,1)$, respectively. Agent $i$ 's program can be written

$$
\begin{aligned}
v_{i}= & \max _{x_{i} \geqslant 0}\left\{\lambda\left[\alpha_{i}\left(1-w_{i}\right)+\sum_{j \neq i} \alpha_{j} \psi_{j i} \delta v_{i}\right]\right. \\
& \left.+(1-\lambda)\left[p_{i}(\mathbf{x})\left(1-w_{i}\right)+\sum_{j \neq i} p_{j}(\mathbf{x}) \psi_{j i} \delta v_{i}\right]-c x_{i}\right\}
\end{aligned}
$$

Differentiating the r.h.s. of (A.16), we obtain

$$
(1-\lambda) \frac{\partial p_{i}(\mathbf{x})}{\partial x_{i}} \pi_{i}^{k}-c \leqslant 0 \quad\left(=0 \text { if } x_{i}>0\right)
$$


where $\pi_{i}^{k} \equiv 1-w_{i}-\frac{\mu_{i}}{1-p_{i}} \delta_{i} v_{i}$ as in the text. As in the proof of Proposition 4, I first characterize the equilibrium. Suppose $\alpha_{i}>\alpha_{j}$ but, on the contrary, $v_{i}<v_{j}$ for some $i, j \in N$.

Case 1: $v_{k}<v_{i}<v_{j}$. Then, $\psi_{l i}=\psi_{m j}=0$ for $l \neq i$ and $m \neq j$, which implies $w_{i}=w_{j}=w_{k}$ and $\pi_{i}^{k}=\pi_{j}^{k}=1-w_{k}$. From (A.17), we have $x_{i}=x_{j}$ and $p_{i}=p_{j}$. Inserting these facts into (A.16) reveals $v_{i} \geqslant v_{j}$, a contradiction.

Case 2: $v_{i}<v_{j}<v_{k}$. Then, $\psi_{l i}=\psi_{m j}=1$ and hence $\mu_{i}=1-p_{i}$ and $\mu_{j}=1-p_{j}$, revealing that $\pi_{i}^{k}=\pi_{j}^{k}=1-w_{k}-\delta v_{k}$. Again, from (A.17), we have $x_{i}=x_{j}$ and $p_{i}=p_{j}$. Inserting these facts into (A.16) reveals $v_{i} \geqslant v_{j}$, a contradiction.

Case 3: $v_{i} \leqslant v_{k} \leqslant v_{j}$ (at least one inequality being strict). Since $\delta v_{i}<\delta v_{j}$, Lemma 2 implies $\pi_{i}^{k} \leqslant \pi_{j}^{k}$. Given $\pi_{i}^{k} \leqslant \pi_{j}^{k}$, Lemma A2 reveals that $x_{i} \leqslant x_{j}$. This means $p_{i} \leqslant p_{j}$ and $\varepsilon_{i} \geqslant \varepsilon_{j}$. Note that $x_{j}>0$. Otherwise, we would have $x_{j}=x_{i}=0$, which would imply $v_{i} \geqslant v_{j}$, and contradict $v_{i}<$ $v_{j}$. Moreover, using the FOCs in (11), we obtain

$$
\varepsilon_{i} \frac{f\left(x_{i}\right) / x_{i}}{\sum_{l} f\left(x_{l}\right)}\left(1-p_{i}\right) \pi_{i}^{k} \leqslant c=\varepsilon_{j} \frac{f\left(x_{j}\right) / x_{j}}{\sum_{l} f\left(x_{l}\right)}\left(1-p_{j}\right) \pi_{j}^{k} .
$$

Since $f\left(x_{i}\right) / x_{i} \leqslant f\left(x_{j}\right) / x_{j}$ and $\varepsilon_{i} \geqslant \varepsilon_{j}$, we have $\left(1-p_{i}\right) \pi_{i}^{k} \leqslant\left(1-p_{j}\right) \pi_{j}^{k}$. Moreover, since $\varepsilon_{i} p_{i}(1-$ $\left.p_{i}\right)=-\left(1-p_{i}\right)+1-\bar{p}_{i}$ by definition, we also have

$$
\left[-\left(1-p_{i}\right)+1-\bar{p}_{i}\right] \pi_{i}^{k} \leqslant c x_{i} \leqslant c x_{j}=\left[-\left(1-p_{j}\right)+1-\bar{p}_{j}\right] \pi_{j}^{k}
$$

and hence $\left(1-\bar{p}_{i}\right) \pi_{i}^{k} \leqslant\left(1-\bar{p}_{j}\right) \pi_{j}^{k}$.

Next we combine (A.16) and (A.17), to obtain a similar expression to Eq. (A.7):

$$
v_{i}=1-w_{i}-R_{i},
$$

where $R_{i} \equiv \lambda\left(1-\alpha_{i}\right) \tilde{\pi}_{i}^{k}+(1-\lambda)\left(1-\bar{p}_{i}\right) \pi_{i}^{k}, \tilde{\pi}_{i}^{k} \equiv 1-w_{i}-\frac{\widetilde{\mu}_{i}}{1-\alpha_{i}} \delta_{i} v_{i}$, and $\tilde{\mu}_{i} \equiv \sum_{j \neq i} \alpha_{j} \psi_{j i}$.

Eq. (A.5) reveals that $w_{i}=w_{k}+\delta v_{k}-\delta v_{i}$ and $w_{j}=w_{k}$. Furthermore, from (A.18), we have $v_{i}=\frac{1-w_{k}-\delta v_{k}-R_{i}}{1-\delta}$ and $v_{j}=1-w_{k}-R_{j}$, respectively. Since $v_{i} \leqslant v_{k}$ by hypothesis, it follows:

$$
\begin{aligned}
\frac{1-w_{k}-\delta v_{k}-R_{i}}{1-\delta} & \leqslant v_{k} \\
& \Longrightarrow 1-w_{k}-R_{i} \leqslant v_{k} .
\end{aligned}
$$

Moreover, given $v_{k} \leqslant v_{j}$, (A.19) implies $1-w_{k}-R_{i}<1-w_{k}-R_{j}$, where the strict inequality is due to the hypothesis of Case 3. From here, we have $R_{j}<R_{i}$, or equivalently

$$
\lambda\left(1-\alpha_{j}\right) \tilde{\pi}_{j}^{k}+(1-\lambda)\left(1-\bar{p}_{j}\right) \pi_{j}^{k}<\lambda\left(1-\alpha_{i}\right) \tilde{\pi}_{i}^{k}+(1-\lambda)\left(1-\bar{p}_{i}\right) \pi_{i}^{k} .
$$

Since $\left(1-\bar{p}_{i}\right) \pi_{i}^{k} \leqslant\left(1-\bar{p}_{j}\right) \pi_{j}^{k}$, it must be that $\left(1-\alpha_{j}\right) \tilde{\pi}_{j}^{k}<\left(1-\alpha_{i}\right) \tilde{\pi}_{i}^{k}$. Moreover, using the definitions of $\tilde{\pi}_{i}^{k}$ and $\widetilde{\pi}_{j}^{k}$, and recalling $\alpha_{i}>\alpha_{j}$, it follows that $\tilde{\mu}_{i}<\tilde{\mu}_{j}$. However, since $v_{i}<v_{j}$, we have $\tilde{\mu}_{i} \geqslant \tilde{\mu}_{j}$, a contradiction.

Overall, these three cases reveal that $v_{i} \geqslant v_{j}$, which, given $\delta v_{i} \geqslant \delta v_{j}$ and Lemma 2, implies $\pi_{i}^{k} \geqslant \pi_{j}^{k}$. From (A.17), this further implies $x_{i} \geqslant x_{j}$. The existence and uniqueness of equilibrium pair $(\mathbf{x}, \mathbf{v})$ can be established by using similar arguments in Proposition 4. 


\section{Acknowledgments}

I thank two anonymous referees and the associate editor for very useful comments and suggestions. I also thank Jeremy Burke, Florin Dorobantu, Hulya Eraslan, Wolfgang Kohler, Tracy Lewis, Curtis Taylor, and seminar participants at Bilkent University, Boston College, the 2005 Midwest Theory Conference, the 2005 Public Choice Meetings, and the 2006 Summer Meetings of Econometric Society for helpful comments and discussions. Paul Dudenhefer provided valuable editorial help. Part of this research was undertaken when I visited the Kellogg School of Management. I am grateful for its hospitality. All remaining errors are mine.

\section{References}

[1] D. Baron, J. Ferejohn, Bargaining and agenda control formation in legislatures, Amer. Econ. Rev. 77 (1987) 303-309 (Papers and Proceedings).

[2] D. Baron, J. Ferejohn, Bargaining in legislatures, Amer. Polit. Sci. Rev. 83 (1989) 1181-1206.

[3] D. Baron, J. Ferejohn, The power to propose, in: P.C. Ordeshook (Ed.), Models of Strategic Choice in Politics, University of Michigan Press, Ann Arbor, 1989, pp. 343-366.

[4] D. Baron, E. Kalai, The simplest equilibrium of a majority-rule division game, J. Econ. Theory 61 (1993) $290-301$.

[5] K. Binmore, Perfect equilibria in bargaining models, in: K. Binmore, P. Dasgupta (Eds.), The Economics of Bargaining, Basil Blackwell, Oxford, 1987, pp. 77-105.

[6] F. Bloch, Sequential formation of coalitions with fixed payoff division, Games Econ. Behav. 14 (1996) 90-123.

[7] F. Bloch, S.S. Pages, R. Soubeyran, When does universal peace prevail? Secession and group formation in rentseeking contests and policy conflicts, Econ. Governance 7 (2006) 3-29.

[8] K. Chatterjee, B. Dutta, D. Ray, K. Sengupta, A noncooperative theory of coalitional bargaining, Rev. Econ. Stud. 60 (1993) 463-477.

[9] Y.K. Che, I. Gale, Caps on political lobbying, Amer. Econ. Rev. 88 (1998) 643-651.

[10] H. Eraslan, Uniqueness of stationary equilibrium payoffs in the Baron-Ferejohn model, J. Econ. Theory 103 (2002) $11-30$.

[11] H. Eraslan, A. Merlo, Majority rule in a stochastic model of bargaining, J. Econ. Theory 103 (2002) $31-48$.

[12] J. Esteban, D. Ray, Conflict and distribution, J. Econ. Theory 87 (1999) 379-415.

[13] R. Evans, Coalitional bargaining with competition to make offers, Games Econ. Behav. 19 (1997) 211-220.

[14] G.W. Fairholm, Organizational Power Politics: Tactics in Organizational Leadership, Praeger, Westport, CT, 1993.

[15] F. Gul, Bargaining foundations of the Shapley value, Econometrica 57 (1989) 81-95.

[16] J. Harrington, The power of the proposal maker in a model of endogenous agenda formation, Public Choice 94 (1990) $1-20$.

[17] M. Herrero, A strategic bargaining approach to market institutions, Ph.D. Thesis, University of London, 1985.

[18] R. Inderst, H. Muller, K. Warneryd, Distributional conflict in organizations, Europ. Econ. Rev., in press.

[19] B. Knight, Estimating the value of proposal power, Amer. Econ. Rev. 95 (2005) 1639-1652.

[20] W. Leininger, More efficient rent-seeking — a Munchhausen solution, Public Choice 75 (1993) $43-62$.

[21] E. Maskin, Bargaining, coalitions, and externalities, Working Paper, Princeton University, 2003.

[22] A. Merlo, C. Wilson, A stochastic model of sequential bargaining with complete information, Econometrica 63 (1995) 371-399.

[23] A. Merlo, C. Wilson, Efficient delays in a stochastic model of bargaining, Econ. Theory 11 (1998) 39-55.

[24] H. Muller, K. Warneryd, Inside versus outside ownership: a political theory of firm, RAND J. Econ. 32 (2001) 527-541.

[25] S. Nitzan, Modeling rent-seeking contests, Europ. J. Polit. Economy 10 (1994) 41-60.

[26] K.O. Nti, Rent-seeking with asymmetric valuations, Public Choice 98 (1999) 415-430.

[27] A. Okada, A noncooperative coalitional bargaining game with random proposers, Games Econ. Behav. 16 (1996) 97-108.

[28] D. Perez-Castrillo, D. Wettstein, Choosing wisely: a multibidding approach, Amer. Econ. Rev. 92 (2002) $1577-1587$.

[29] D. Perez-Castrillo, D. Wettstein, An ordinal Shapley value for economic environments, J. Econ. Theory 127 (2006) 296-308.

[30] J. Pfeffer, Power in Organizations, Pittman, Marshfield, MA, 1981.

[31] D. Ray, R. Vohra, Equilibrium binding agreements, J. Econ. Theory 73 (1997) 30-78. 
[32] D. Ray, R. Vohra, A theory of endogenous coalition structures, Games Econ. Behav. 26 (1999) 286-336.

[33] A. Rubinstein, Perfect equilibrium in a bargaining model, Econometrica 50 (1982) 97-109.

[34] S. Skaperdas, Cooperation, conflict, and power in the absence of property rights, Amer. Econ. Rev. 82 (1992) $720-739$.

[35] S. Skaperdas, Contest success functions, Econ. Theory 7 (1996) 283-290.

[36] J. Sutton, Noncooperative bargaining theory: an introduction, Rev. Econ. Stud. 53 (1986) 245-262.

[37] F. Szidarovski, K. Okuguchi, On the existence and uniqueness of pure Nash equilibrium in rent-seeking games, Games Econ. Behav. 18 (1997) 135-140.

[38] E. Winter, Incentives and discrimination, Amer. Econ. Rev. 94 (2004) 764-773. 